\title{
SOLAR SPECTRAL IRRADIANCE CHANGES DURING CYCLE 24
}

\author{
S. V. Marchenko ${ }^{1}$ and M. T. DeLand ${ }^{1}$ \\ Science Systems and Applications, Inc., Lanham, MD, USA \\ Received 2014 January 23; accepted 2014 May 22; published 2014 June 20
}

\begin{abstract}
We use solar spectra obtained by the Ozone Monitoring Instrument (OMI) on board the Aura satellite to detect and follow long-term (years) and short-term (weeks) changes in the solar spectral irradiance (SSI) in the 265-500 nm spectral range. During solar Cycle 24, in the relatively line-free regions the SSI changed by $\sim 0.6 \% \pm 0.2 \%$ around $265 \mathrm{~nm}$. These changes gradually diminish to $0.15 \% \pm 0.20 \%$ at $500 \mathrm{~nm}$. All strong spectral lines and blends, with the notable exception of the upper Balmer lines, vary in unison with the solar "continuum." Besides the lines with strong chromospheric components, the most involved species include $\mathrm{Fe}$ I blends and all prominent $\mathrm{CH}$, $\mathrm{NH}$, and $\mathrm{CN}$ spectral bands. Following the general trend seen in the solar "continuum," the variability of spectral lines also decreases toward longer wavelengths. The long-term solar cycle SSI changes are closely, to within the quoted $0.1 \%-0.2 \%$ uncertainties, matched by the appropriately adjusted short-term SSI variations derived from the 27 day rotational modulation cycles. This further strengthens and broadens the prevailing notion about the general scalability of the UV SSI variability to the emissivity changes in the Mg II $280 \mathrm{~nm}$ doublet on timescales from weeks to years. We also detect subtle deviations from this general rule: the prominent spectral lines and blends at $\lambda \gtrsim 350 \mathrm{~nm}$ show slightly more pronounced 27 day SSI changes when compared to the long-term (years) trends. We merge the solar data from Cycle 21 with the current Cycle 24 OMI and GOME-2 observations and provide normalized SSI variations for the 170-795 nm spectral region.
\end{abstract}

Key words: Sun: activity - Sun: atmosphere - Sun: rotation - Sun: UV radiation

Online-only material: machine-readable table

\section{INTRODUCTION}

The variability of solar output on a wide range of temporal and spectral scales is well known. A succession of steadily improving instruments and measurements have now established that the solar cycle variation of total solar irradiance (TSI) is on the order of $\sim 0.1 \%$, with a more precise value depending on the choice of data set and solar cycle (e.g., Fröhlich 2009; Kopp $\&$ Lean 2011). The anticipated relatively low amplitude of the solar spectral irradiance (SSI) variability at longer $(\lambda \gtrsim 350 \mathrm{~nm})$ wavelengths imposes severe constraints on the accuracy and temporal stability of instruments. At shorter wavelengths the SSI changes can be revealed almost exclusively via satellite measurements, which introduces additional complications due to the effects of relatively rapid instrument degradation (e.g., DeLand \& Cebula 2012; Ermolli et al. 2013). An alternative approach to estimating long-term solar UV variability is to use a proxy activity index, such as $10.7 \mathrm{~cm}$ flux (Tapping 1987) or the Mg II index (Heath \& Schlesinger 1986) to establish time-dependent variations, coupled with wavelength-dependent scaling factors that relate proxy variations to irradiance changes. Heath \& Schlesinger (1986) developed scale factors for the spectral range 170-400 nm based on short-term (rotational modulation) variations in Nimbus-7 SBUV irradiance data. DeLand \& Cebula (1993) extended this approach to similar SBUV/2 instruments flying on NOAA spacecraft, and created a composite scale-factors data set. This technique bypasses the issue of instrument degradation corrections that is so important for direct irradiance measurements, but does raise the question of whether long-term solar variations have the same spectral dependence as rotational variations. DeLand \& Cebula (2008) found good agreement between scale-factor

\footnotetext{
1 Also at NASA/Goddard Space Flight Center, Greenbelt, MD, USA.
}

predictions and observed irradiance changes from most of the long-term observations during 1978-2005. We return to this subject, discussing it along with uncertainties in SSI variations, in Section 3.1.

The SORCE satellite was developed to provide a comprehensive measure of solar activity from a single satellite. Launched in 2003 January, it carries three instruments to monitor SSI continuously from X-rays to the near-IR, as well as a TSI instrument (Rottman 2005). SSI variations reported from the Spectral Irradiance Monitor (SIM) measurements by Harder et al. (2009) differ significantly in both magnitude and spectral dependence from most previously reported results. Evaluating the accuracy of the published SIM results is an important issue for understanding solar forcing on climate (Lean \& DeLand 2012; Thuillier et al. 2014), particularly at $\lambda>300 \mathrm{~nm}$ where previous satellite and ground-based measurements provide relatively coarse upper limits to long-term variations (Thuillier et al. 2004). Combining the contemporaneous Ozone Monitoring Instrument (OMI) and GOME-2 observations with the records from previous solar cycles, we construct the normalized (to the $\mathrm{Mg}$ II doublet at $280 \mathrm{~nm}$ ) solar variability spectrum in the 170-795 nm spectral range, which could be used in combination with a reference spectrum (e.g., Thuillier et al. 2004) to reproduce a dynamical solar spectrum at any epoch.

\section{THE DATA}

The remote-sensing OMI (part of the Aura instrumental suite; Levelt et al. 2006) has collected information about trace gases in the Earth's atmosphere since 2004 July. OMI comprises three different spectral channels, UV1 (264-311 nm spectral domain, resolution $\delta \lambda=0.63 \mathrm{~nm})$, UV2 $(307-383 \mathrm{~nm}, \delta \lambda=0.42 \mathrm{~nm})$, and VIS (349-504 nm, $\delta \lambda=0.63 \mathrm{~nm}$ ), each acquiring data through partially shared optical pathways (Dobber et al. 2006). The UV1 and UV2 light falls on the same CCD detector 


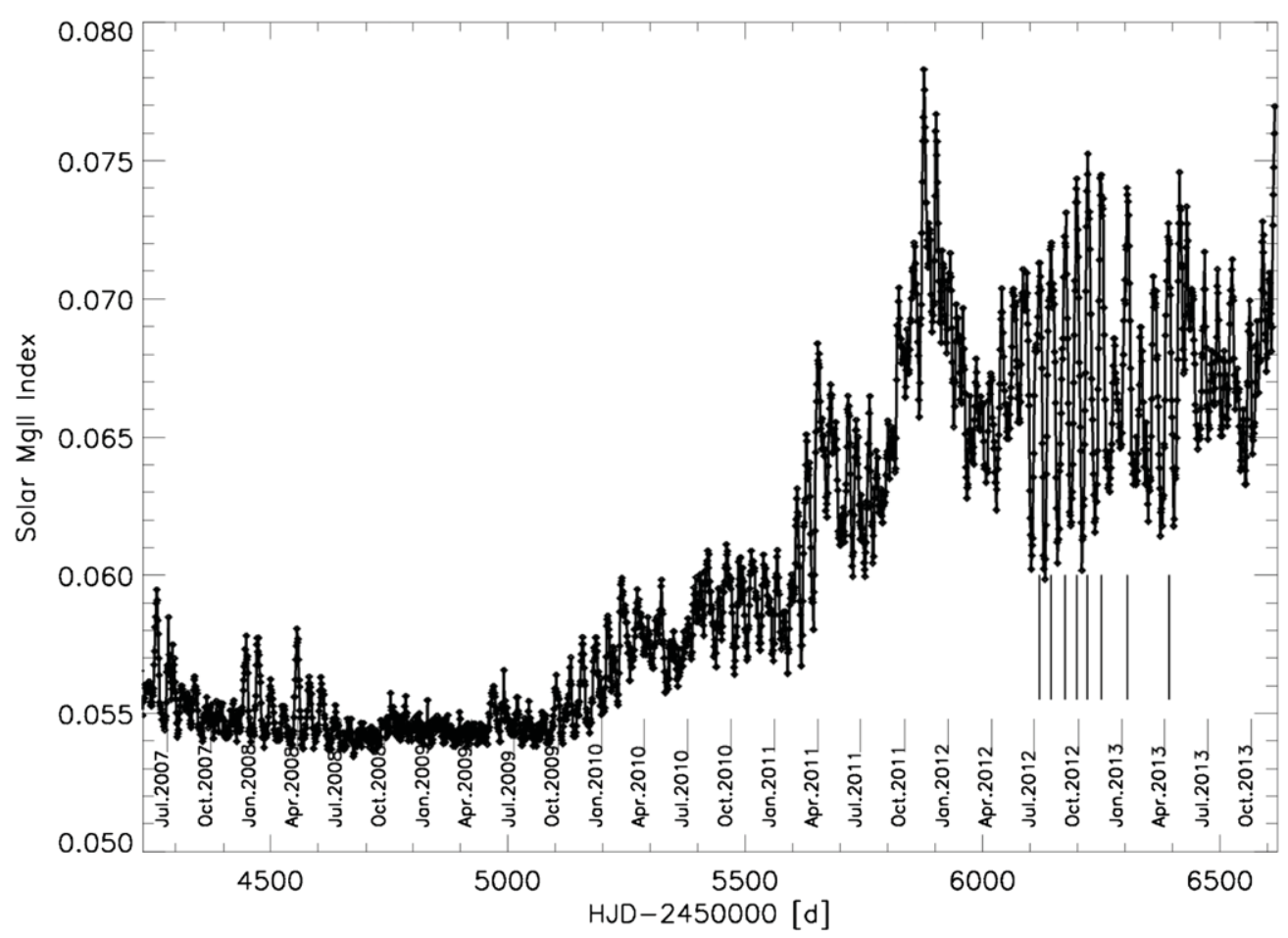

Figure 1. OMI Mg II index (see DeLand \& Marchenko 2013) for Cycle 23 and Cycle 24, scaled to the SORCE SOLSTICE data (see DeLand \& Marchenko 2013 for more details), with the 27 day rotational cycles used to create an average max-min difference spectrum marked by vertical lines.

while VIS spectra are recorded by a different CCD. The broad field of view provides either 30 (UV1) or 60 (UV2, VIS) simultaneously recorded spectra (we call them rows in the forthcoming discussion) for a single two-second image, with each row binned on a slightly different wavelength grid. This "spectral smile" effect may produce highly oversampled combined (by adding different rows) daily solar spectrum that improves our ability to identify and monitor solar absorption features. OMI observes the Sun on a daily basis. The daily solar irradiances are routinely used for calibration purposes. The solar light and the back-scattered Earth-shine go through a similar optical pathway, save three elements: the primary mirror, the folding mirror, and the solar diffusers. The use of the triplediffuser approach with different duty cycles, the volume diffuser for daily observations and two aluminum reflective diffusors as weekly and monthly controlling devices, enables us to quantify the degradation rates in the irradiance pathway. ${ }^{2}$

A thorough understanding of both instrument stability (e.g., wavelength shifts, stray light contamination) and long-term sensitivity changes are obviously essential for the interpretation of solar irradiance measurements, particularly in the near-UV and visible regions where irradiance variations are small. We provide an extensive discussion of our analysis for these topics in Appendices A and B. All OMI results presented in the following sections of this paper include the best corrections for derived instrumental trends.

\subsection{The Long-term Maximum-Minimum Difference Spectrum}

We apply the degradation model (see Appendix B) to the monthly averages of solar irradiances. The small, $\lesssim 0.005 \mathrm{~nm}$, wavelength shifts (see Appendix A) between different epochs

\footnotetext{
See some relevant diagnostics at

http://www.knmi.nl/omi/research/calibration/instrument_status_v3/index.html.
}

are adjusted accordingly. In order to minimize the possible instrumental biases related to the gradual seasonally changing incidence angles of solar light illuminating the solar diffuser (for short solar goniometry), we create pair-wise differences (e.g., 2013 August-2008 August) between each monthly average solar spectrum and the corresponding "reference" monthly average spectra obtained around the solar minimum (for the purpose of this study defined as the time period $4300<t<$ 5100 days, see Figure 1, where the times are expressed as HJD-2450000), thus sampling the period from early 2007 until late 2013. We average the corresponding differences created for different "reference" years, thus benefiting from the unusually prolonged solar minimum; e.g., we combine (2013 August-2007 August) with (2013 August-2008 August) and (2013 August-2009 August). Each pair-wise difference is normalized to the relevant minimum spectrum before the final time-averaging. In order to reveal the SSI changes in the ongoing cycle 24 , we spectrally bin the differences around wavelengths centered either on strong spectral features or on the regions relatively free from spectral lines. Table 1 lists the wavelength-averaged, normalized long-term difference spectra in percentages for prominent spectral lines or blends. We provide the central and then starting and finishing wavelengths for each bin; the times are given as HJD-2450000. Table 2 has a similar format and provides the amplitudes of changes for the "continuum" regions. The wavelength-binned difference timeseries are plotted in Figure 2 along with the normalized $\mathrm{Mg}$ II index from DeLand \& Marchenko (2013). We note the close match between the newly derived estimates of the variable flux at the $\mathrm{Mg}$ II doublet and the traditional $\mathrm{Mg}$ II index. Both data sets come from the same instrument, however, using profoundly different evaluation techniques. The currently implemented approach is based on the self-reliant (see Appendix B) OMI degradation model, while the Mg II index, which is inherently less prone to the instrumental degradation, was further refined 
Table 1

The Wavelength-averaged, Long-term SSI Changes for Prominent Spectral Lines and Blends

\begin{tabular}{|c|c|c|c|c|c|c|c|c|c|c|c|c|}
\hline$\ldots$ & $272.00^{\mathrm{a}}$ & 274.60 & 280.00 & 285.25 & 288.20 & 300.20 & 358.50 & 383.00 & 393.50 & 397.00 & 430.50 & 486.15 \\
\hline$\ldots$ & $271.60^{b}$ & 274.00 & 279.50 & 285.00 & 287.90 & 299.20 & 357.50 & 381.00 & 393.25 & 396.75 & 429.50 & 485.90 \\
\hline$\ldots$ & $272.40^{\mathrm{c}}$ & 275.20 & 280.50 & 285.50 & 288.50 & 301.20 & 359.50 & 385.00 & 393.75 & 397.25 & 431.50 & 486.40 \\
\hline $4115.50^{d}$ & $0.01^{\mathrm{e}}$ & 0.04 & 0.64 & 0.09 & -0.04 & -0.07 & -0.09 & 0.02 & 0.11 & 0.07 & 0.01 & -0.03 \\
\hline 4146.50 & -0.01 & 0.01 & 0.45 & 0.01 & -0.01 & -0.07 & -0.08 & 0.01 & 0.08 & 0.06 & 0.00 & -0.04 \\
\hline 4174.50 & -0.01 & 0.01 & 0.28 & 0.00 & -0.02 & -0.05 & -0.06 & 0.02 & 0.04 & 0.04 & 0.01 & 0.00 \\
\hline 4205.50 & -0.05 & -0.01 & 0.09 & -0.05 & -0.05 & -0.07 & -0.07 & 0.01 & 0.04 & 0.03 & -0.00 & -0.02 \\
\hline 4235.50 & -0.13 & -0.08 & 0.20 & -0.09 & -0.12 & -0.15 & -0.11 & -0.02 & 0.04 & 0.02 & -0.01 & -0.03 \\
\hline 4266.50 & -0.05 & -0.03 & 0.33 & 0.00 & -0.04 & -0.06 & -0.06 & 0.03 & 0.08 & 0.05 & 0.02 & -0.01 \\
\hline 4296.50 & -0.02 & 0.02 & 0.46 & 0.03 & -0.03 & -0.05 & -0.04 & 0.05 & 0.11 & 0.09 & 0.06 & 0.06 \\
\hline 4327.50 & -0.06 & -0.03 & 0.37 & -0.01 & -0.07 & -0.08 & -0.08 & 0.00 & 0.04 & 0.01 & 0.00 & -0.03 \\
\hline 4358.50 & -0.10 & -0.06 & 0.03 & -0.10 & -0.10 & -0.09 & -0.07 & -0.00 & -0.04 & 0.02 & -0.00 & -0.02 \\
\hline 4388.50 & -0.21 & -0.18 & -0.14 & -0.24 & -0.18 & -0.17 & -0.04 & 0.00 & -0.01 & 0.02 & -0.00 & -0.06 \\
\hline 4480.50 & -0.11 & -0.09 & -0.24 & -0.23 & -0.08 & -0.07 & -0.07 & -0.01 & -0.06 & -0.03 & -0.02 & -0.03 \\
\hline 4511.50 & -0.14 & -0.14 & -0.01 & -0.18 & -0.19 & -0.13 & -0.09 & -0.05 & -0.04 & -0.08 & -0.06 & -0.08 \\
\hline 4540.50 & -0.05 & -0.03 & 0.06 & -0.13 & -0.04 & -0.03 & -0.08 & -0.02 & 0.02 & 0.01 & -0.04 & -0.04 \\
\hline 4571.50 & -0.06 & -0.04 & 0.09 & -0.06 & -0.09 & -0.05 & -0.06 & -0.02 & -0.01 & -0.04 & -0.03 & -0.05 \\
\hline 4601.50 & -0.19 & -0.19 & -0.27 & -0.33 & -0.24 & -0.17 & -0.09 & -0.05 & -0.06 & -0.06 & -0.02 & -0.04 \\
\hline 4632.50 & -0.06 & -0.09 & -0.21 & -0.19 & -0.10 & -0.07 & -0.07 & -0.04 & -0.06 & -0.05 & -0.03 & 0.00 \\
\hline 4662.50 & 0.03 & -0.00 & -0.25 & -0.06 & 0.01 & 0.04 & 0.02 & -0.00 & -0.09 & -0.07 & 0.01 & 0.00 \\
\hline 4693.50 & -0.07 & -0.05 & -0.29 & -0.14 & -0.11 & -0.03 & 0.01 & -0.02 & -0.08 & -0.05 & -0.03 & -0.02 \\
\hline 4724.50 & -0.05 & -0.04 & -0.13 & -0.11 & -0.04 & -0.01 & 0.01 & -0.02 & -0.04 & -0.05 & -0.02 & -0.03 \\
\hline 4754.50 & 0.20 & 0.18 & 0.14 & 0.14 & 0.12 & 0.17 & 0.05 & 0.00 & -0.02 & -0.05 & -0.00 & 0.02 \\
\hline 4846.50 & 0.03 & 0.02 & -0.08 & 0.05 & -0.04 & 0.01 & 0.02 & -0.02 & -0.08 & -0.08 & -0.02 & -0.03 \\
\hline 4877.50 & 0.15 & 0.13 & 0.02 & 0.14 & 0.11 & 0.12 & 0.09 & 0.05 & 0.00 & 0.03 & 0.05 & 0.04 \\
\hline 4905.50 & 0.05 & 0.03 & -0.06 & 0.05 & -0.02 & 0.02 & 0.08 & 0.02 & -0.06 & -0.05 & 0.03 & 0.01 \\
\hline 4936.50 & 0.08 & 0.04 & -0.08 & 0.02 & 0.04 & 0.04 & 0.06 & 0.02 & -0.02 & 0.01 & 0.02 & 0.01 \\
\hline 4966.50 & 0.19 & 0.18 & 0.28 & 0.29 & 0.17 & 0.17 & 0.08 & 0.05 & 0.03 & 0.03 & 0.01 & 0.00 \\
\hline 4997.50 & 0.08 & 0.09 & 0.22 & 0.14 & 0.05 & 0.07 & 0.07 & 0.04 & 0.04 & 0.03 & 0.02 & -0.02 \\
\hline 5027.50 & -0.01 & -0.04 & -0.17 & -0.01 & -0.10 & -0.00 & 0.01 & -0.05 & -0.09 & -0.09 & -0.09 & -0.17 \\
\hline 5058.50 & 0.11 & 0.10 & -0.02 & 0.16 & 0.05 & 0.09 & 0.08 & 0.02 & -0.01 & -0.00 & 0.01 & -0.01 \\
\hline 5089.50 & 0.15 & 0.10 & 0.18 & 0.13 & 0.01 & 0.09 & 0.06 & 0.02 & 0.04 & -0.02 & 0.01 & -0.01 \\
\hline 5119.50 & 0.16 & 0.12 & 0.29 & 0.03 & -0.03 & 0.11 & 0.01 & -0.01 & -0.02 & -0.03 & -0.01 & -0.03 \\
\hline 5211.50 & 0.19 & 0.19 & 0.75 & 0.29 & 0.06 & 0.12 & 0.10 & 0.05 & 0.12 & 0.08 & 0.04 & -0.01 \\
\hline 5242.50 & 0.28 & 0.29 & 1.45 & 0.54 & 0.17 & 0.22 & 0.14 & 0.12 & 0.25 & 0.19 & 0.08 & 0.03 \\
\hline 5270.50 & 0.32 & 0.32 & 1.27 & 0.50 & 0.18 & 0.21 & 0.16 & 0.11 & 0.25 & 0.19 & 0.08 & 0.04 \\
\hline 5301.50 & 0.26 & 0.26 & 1.11 & 0.45 & 0.17 & 0.18 & 0.12 & 0.09 & 0.22 & 0.18 & 0.07 & 0.03 \\
\hline 5331.50 & 0.15 & 0.13 & 0.86 & 0.30 & 0.04 & 0.08 & 0.09 & 0.06 & 0.15 & 0.10 & 0.05 & -0.01 \\
\hline 5362.50 & 0.24 & 0.21 & 0.86 & 0.33 & 0.12 & 0.15 & 0.10 & 0.07 & 0.17 & 0.12 & 0.05 & 0.01 \\
\hline 5392.50 & 0.36 & 0.33 & 1.31 & 0.58 & 0.25 & 0.25 & 0.19 & 0.13 & 0.28 & 0.20 & 0.09 & 0.04 \\
\hline 5423.50 & 0.31 & 0.27 & 1.37 & 0.56 & 0.18 & 0.21 & 0.13 & 0.09 & 0.27 & 0.18 & 0.06 & 0.00 \\
\hline 5454.50 & 0.33 & 0.35 & 1.73 & 0.65 & 0.17 & 0.23 & 0.16 & 0.13 & 0.34 & 0.24 & 0.08 & 0.01 \\
\hline 5484.50 & 0.30 & 0.30 & 1.70 & 0.64 & 0.23 & 0.19 & 0.19 & 0.15 & 0.34 & 0.23 & 0.10 & 0.03 \\
\hline 5576.50 & 0.28 & 0.25 & 1.35 & 0.57 & 0.10 & 0.19 & 0.14 & 0.08 & 0.22 & 0.15 & 0.05 & -0.01 \\
\hline 5607.50 & 0.38 & 0.37 & 1.89 & 0.80 & 0.17 & 0.24 & 0.14 & 0.09 & 0.33 & 0.21 & 0.04 & -0.03 \\
\hline 5635.50 & 0.50 & 0.50 & 2.96 & 1.03 & 0.35 & 0.31 & 0.20 & 0.16 & 0.51 & 0.39 & 0.10 & 0.01 \\
\hline 5666.50 & 0.64 & 0.68 & 3.75 & 1.32 & 0.42 & 0.38 & 0.29 & 0.24 & 0.75 & 0.52 & 0.14 & 0.03 \\
\hline 5696.50 & 0.52 & 0.53 & 2.76 & 1.00 & 0.30 & 0.32 & 0.22 & 0.20 & 0.56 & 0.40 & 0.14 & 0.03 \\
\hline 5727.50 & 0.68 & 0.68 & 3.21 & 1.23 & 0.49 & 0.43 & 0.26 & 0.24 & 0.64 & 0.46 & 0.17 & 0.07 \\
\hline 5757.50 & 0.69 & 0.65 & 2.31 & 1.08 & 0.48 & 0.46 & 0.28 & 0.23 & 0.55 & 0.39 & 0.18 & 0.09 \\
\hline 5788.50 & 0.54 & 0.59 & 2.91 & 1.12 & 0.34 & 0.37 & 0.23 & 0.20 & 0.57 & 0.42 & 0.12 & 0.00 \\
\hline 5819.50 & 0.67 & 0.74 & 4.26 & 1.44 & 0.41 & 0.42 & 0.26 & 0.25 & 0.80 & 0.58 & 0.15 & -0.01 \\
\hline 5849.50 & 0.81 & 0.83 & 4.75 & 1.72 & 0.46 & 0.47 & 0.31 & 0.32 & 0.97 & 0.71 & 0.21 & 0.01 \\
\hline 5941.50 & 0.83 & 0.85 & 4.63 & 1.66 & 0.55 & 0.52 & 0.33 & 0.32 & 0.90 & 0.63 & 0.21 & 0.03 \\
\hline 5972.50 & 0.67 & 0.68 & 3.51 & 1.35 & 0.41 & 0.44 & 0.29 & 0.26 & 0.70 & 0.51 & 0.17 & 0.02 \\
\hline 6001.50 & 0.72 & 0.72 & 3.74 & 1.38 & 0.51 & 0.47 & 0.29 & 0.26 & 0.72 & 0.48 & 0.17 & 0.03 \\
\hline 6032.50 & 0.75 & 0.77 & 3.95 & 1.45 & 0.53 & 0.48 & 0.31 & 0.27 & 0.78 & 0.56 & 0.18 & 0.03 \\
\hline 6062.50 & 0.75 & 0.80 & 3.99 & 1.55 & 0.48 & 0.45 & 0.25 & 0.27 & 0.84 & 0.58 & 0.19 & 0.02 \\
\hline 6093.50 & 0.86 & 0.93 & 4.94 & 1.81 & 0.59 & 0.53 & 0.34 & 0.30 & 0.82 & 0.59 & 0.23 & 0.10 \\
\hline 6123.50 & 0.94 & 0.99 & 4.96 & 1.86 & 0.69 & 0.57 & 0.33 & 0.30 & 0.89 & 0.65 & 0.21 & 0.08 \\
\hline 6154.50 & 0.94 & 1.00 & 5.08 & 1.94 & 0.71 & 0.63 & 0.37 & 0.32 & 0.90 & 0.64 & 0.23 & 0.06 \\
\hline 6185.50 & 0.96 & 0.99 & 5.32 & 1.91 & 0.70 & 0.61 & 0.36 & 0.34 & 0.96 & 0.69 & 0.25 & 0.05 \\
\hline 6215.50 & 1.03 & 1.06 & 4.60 & 1.90 & 0.68 & 0.58 & 0.43 & 0.38 & 0.98 & 0.68 & 0.29 & 0.09 \\
\hline 6307.50 & 0.93 & 0.91 & 4.46 & 1.68 & 0.58 & 0.55 & 0.38 & 0.33 & 0.85 & 0.63 & 0.24 & 0.06 \\
\hline 6338.50 & 0.75 & 0.77 & 3.51 & 1.43 & 0.51 & 0.50 & 0.38 & 0.29 & 0.73 & 0.50 & 0.22 & 0.07 \\
\hline
\end{tabular}


Table 1

(Continued)

\begin{tabular}{lcccccccccccc}
\hline$\ldots$ & $272.00^{\mathrm{a}}$ & 274.60 & 280.00 & 285.25 & 288.20 & 300.20 & 358.50 & 383.00 & 393.50 & 397.00 & 430.50 & 486.15 \\
\hline 6366.50 & 0.86 & 0.86 & 4.01 & 1.52 & 0.58 & 0.54 & 0.39 & 0.32 & 0.80 & 0.59 & 0.25 \\
6397.50 & 0.99 & 1.14 & 5.64 & 2.19 & 0.87 & 0.64 & 0.40 & 0.34 & 0.98 & 0.68 & 0.25 \\
6427.50 & 1.05 & 1.12 & 5.46 & 2.11 & 0.67 & 0.62 & 0.41 & 0.40 & 1.13 & 0.77 & 0.29 \\
6458.50 & 1.01 & 1.05 & 4.35 & 1.81 & 0.74 & 0.65 & 0.42 & 0.38 & 0.95 & 0.67 & 0.29 \\
6488.50 & 1.11 & 1.10 & 4.48 & 1.88 & 0.84 & 0.74 & 0.46 & 0.39 & 0.95 & 0.72 & 0.31 \\
6519.50 & 1.01 & 1.06 & 4.67 & 1.97 & 0.73 & 0.67 & 0.44 & 0.38 & 1.01 & 0.71 & 0.28 \\
6550.50 & 0.92 & 0.92 & 4.00 & 1.69 & 0.64 & 0.61 & 0.38 & 0.34 & 0.83 & 0.60 & 0.27 \\
6580.50 & 0.97 & 0.92 & 4.62 & 1.70 & 0.54 & 0.59 & 0.43 & 0.38 & 0.99 & 0.71 & 0.31 & 0.08 \\
\hline
\end{tabular}

Notes.

${ }^{\text {a }}$ Central wavelength, $\mathrm{nm}$.

${ }^{\mathrm{b}}$ Starting wavelength, $\mathrm{nm}$.

${ }^{\mathrm{c}}$ Finishing wavelength, nm.

d Time, days, as HJD-2450000.

e Normalized differences, $\%$.

Table 2

The Wavelength-averaged, Long-term SSI Changes for Weak Line Blends ${ }^{\mathrm{a}}$

\begin{tabular}{|c|c|c|c|c|c|c|c|c|c|c|c|c|c|c|c|}
\hline$\cdots$ & 265.0 & 268.5 & 283.5 & 292.5 & 304.5 & 312.5 & 327.5 & 341.0 & 367.5 & 402.0 & 442.0 & 457.5 & 470.5 & 482.5 & 499.5 \\
\hline . . & 264.0 & 267.0 & 283.0 & 290.0 & 303.0 & 311.0 & 326.0 & 339.0 & 366.0 & 401.0 & 441.0 & 455.0 & 468.0 & 480.0 & 497.0 \\
\hline$\ldots$ & 266.0 & 270.0 & 284.0 & 295.0 & 306.0 & 314.0 & 329.0 & 343.0 & 369.0 & 403.0 & 443.0 & 460.0 & 473.0 & 485.0 & 502.0 \\
\hline 4115.50 & 0.06 & 0.03 & -0.04 & -0.08 & -0.03 & -0.07 & -0.05 & -0.05 & -0.11 & -0.01 & -0.00 & -0.01 & -0.00 & 0.00 & 0.01 \\
\hline 4146.50 & 0.09 & 0.03 & 0.00 & -0.07 & -0.02 & & -0.04 & -0.04 & & -0.01 & & & -0.00 & -0.00 & 0.01 \\
\hline 4174.50 & 0.07 & 0.04 & -0.00 & -0.04 & 0.01 & -0.01 & -0.00 & -0.01 & -0.07 & 0.01 & 0.01 & 0.01 & 0.02 & 0.02 & 0.04 \\
\hline 4205.50 & 0.04 & 0.02 & -0.02 & -0.05 & -0.00 & & -0.13 & & & & & & 0.01 & & 0.02 \\
\hline 4235.50 & -0.06 & & & & & & & & & & & & -0.02 & & -0.01 \\
\hline 4266.50 & & & -0.02 & -0.0 & -0.03 & -0.02 & -0.01 & -0.01 & -0.09 & -0.00 & & & 0.01 & & 0.02 \\
\hline 4296.50 & 0.09 & 0.07 & -0.02 & -0.05 & 0.00 & 0.02 & 0.03 & 0.03 & -0.05 & 0.03 & 0.07 & 0.07 & 0.08 & 0.10 & 0.12 \\
\hline 4327.50 & 0.03 & -0.01 & -0.05 & -0.09 & -0.04 & -0.01 & -0.00 & -0.00 & -0.09 & -0.01 & 0.00 & -0.01 & -0.01 & -0.00 & 0.01 \\
\hline & -0.01 & -0.04 & -0.04 & -0 . & -0.02 & -0.03 & -0.01 & -0.02 & & -0 & -0.01 & -0.01 & -0.01 & -0.00 & -0.00 \\
\hline 4388.50 & -0.18 & -0.23 & -0.17 & -0.14 & -0.09 & -0.09 & -0.06 & -0.04 & -0.07 & -0.01 & -0.01 & -0.02 & -0.03 & -0.04 & -0.05 \\
\hline 4480.50 & -0.01 & -0.03 & -0.06 & -0.05 & 0.00 & 0.01 & 0.02 & 0.01 & -0.07 & & -0.02 & -0.02 & -0.02 & -0.01 & -0.00 \\
\hline 511.50 & -0.10 & -0.12 & -0.12 & -0.13 & -0.08 & -0.03 & -0.03 & -0.03 & -0.11 & & & -0.07 & -0.07 & -0.06 & -0.07 \\
\hline 540.50 & -0.02 & 0.00 & -0.02 & -0.05 & 0.01 & 0.03 & 0.03 & 0.03 & & & & & -0.04 & -0.02 & -0.03 \\
\hline 4571.50 & -0.04 & -0.03 & -0.03 & -0.05 & 0.00 & 0.02 & 0.02 & 0.02 & -0.08 & & & & -0.04 & -0.03 & -0.03 \\
\hline 4601.50 & -0.14 & -0.16 & -0.16 & -0.16 & -0.09 & -0.03 & -0.01 & -0.01 & -0.08 & & & -0.02 & -0.02 & -0.02 & -0.01 \\
\hline & -0.03 & -0.03 & & & & & 0.02 & & & & & & & & 0.01 \\
\hline & & & & & & & & & & & & & & & 0.04 \\
\hline & -0.0 & & & & & & & & & & & & & & -0.02 \\
\hline & -0.02 & & & & & & & & & & & & & & -0.02 \\
\hline & & & & & & & 0.06 & 0.04 & & & & & 0.03 & 4 & 0.05 \\
\hline & -0.05 & -0.03 & 0.01 & & -0.03 & -0.02 & -0.02 & -0.03 & 0.0 & -0 & & 0.0 & 0.00 & -0 . & -0.02 \\
\hline 877.50 & 0.10 & 0.12 & 0.12 & 0.13 & 0.08 & 0.03 & 0.03 & 0.03 & 0.11 & 8 & 0.08 & 0.08 & 0.07 & 0.06 & 0.07 \\
\hline 905.50 & 0.02 & -0.01 & 0.02 & 0.05 & -0.01 & -0.03 & -0.03 & -0.02 & 0.08 & & 0.06 & 0.06 & 0.04 & 0.02 & 0.03 \\
\hline 936.50 & 0.04 & 0.02 & 0.03 & & -0.00 & -0.02 & -0.02 & -0.02 & 0.08 & & 0.0 & 0.05 & 0.04 & 0.03 & 0.03 \\
\hline 565 & 0.14 & & & & 0.09 & 0.03 & 0.01 & 0.01 & 0.09 & 0.0 & 0.0 & 0.02 & 0.02 & 0.02 & 0.01 \\
\hline 4997.50 & 0.03 & 0.03 & & & 0.04 & 0.00 & -0.02 & -0.01 & 0.08 & 0.03 & 0.01 & 0.01 & 0.00 & 0.00 & -0.00 \\
\hline 5027.50 & -0.17 & -0.12 & -0.07 & -0.02 & -0.04 & -0.07 & -0.09 & -0.08 & 0.03 & -0.05 & -0.10 & -0.11 & -0.13 & -0.15 & -0.18 \\
\hline & & & & & & & -0.01 & & & & & & 0.02 & & 0.01 \\
\hline & & & & & & & & & & & & & 0. & & 0.02 \\
\hline & & & & & & & & & & & & & & & 0.03 \\
\hline 5211.50 & & & & & & & -0. & & & & & & & & 0.03 \\
\hline 5242.50 & 0.20 & 21 & 0.20 & & 0.1 & & 0.05 & 0.05 & 0.12 & & 0.0 & 0.0 & 0.07 & 0.06 & 0.06 \\
\hline 5270.50 & 0.17 & 10 & 0.18 & & & 0.09 & 0.05 & 0.06 & 0.12 & & 0.07 & 0.06 & 0.07 & 0.07 & 0.07 \\
\hline 5301.50 & 0.16 & 0.17 & 0.17 & 0.15 & 0.10 & 0.08 & 0.04 & 0.05 & 0.11 & 0.06 & 0.05 & 0.05 & 0.06 & 0.06 & 0.06 \\
\hline 5331.50 & 0.03 & 0.04 & 0.08 & & 0.00 & 0.02 & -0.01 & 0.00 & 0.08 & 0.03 & 0.04 & 0.04 & 0.04 & 0.04 & 0.03 \\
\hline 5362.50 & 0.12 & & & & & & 0.02 & 0.03 & 0.09 & & 0.05 & 0.05 & 0.05 & 0.05 & 0.05 \\
\hline 5392.50 & 0.23 & & & & & & 0.06 & 0.07 & 0.15 & 0.0 & 0.08 & 0.08 & 0.08 & 0.08 & 0.07 \\
\hline 5423.50 & & & & & & & 0.02 & 0.03 & 0.12 & 0.0 & 0.04 & 0.04 & 0.04 & 0.04 & 0.03 \\
\hline 5454.50 & 0.20 & & 0.20 & & 0.0 & & 0.02 & 0.04 & 0.1 & & 0.05 & 0.04 & 0.05 & 0.04 & 0.04 \\
\hline 5484.50 & & & & & & & & & & & & & 0.09 & & 0.06 \\
\hline 5576.50 & & & & & & & & & & & & & 0.06 & 0.05 & 0.04 \\
\hline 5607.50 & 0.17 & & 0.20 & 0.14 & 0.06 & 0.04 & -0.01 & 0.00 & & & & & 0.04 & 0.03 & 0.01 \\
\hline 5635.50 & 0.28 & 0.28 & 0.27 & 0.20 & 0.12 & 0.11 & 0.04 & 0.05 & 0.13 & 0.03 & 0.05 & 0.04 & 0.05 & 0.05 & 0.04 \\
\hline
\end{tabular}


Table 2

(Continued)

\begin{tabular}{|c|c|c|c|c|c|c|c|c|c|c|c|c|c|c|c|}
\hline$\ldots$ & 265.0 & 268.5 & 283.5 & 292.5 & 304.5 & 312.5 & 327.5 & 341.0 & 367.5 & 402.0 & 442.0 & 457.5 & 470.5 & 482.5 & 499.5 \\
\hline 5666.50 & 0.39 & 0.37 & 0.34 & 0.19 & 0.13 & 0.17 & 0.07 & 0.09 & 0.17 & 0.05 & 0.07 & 0.06 & 0.07 & 0.07 & 0.05 \\
\hline 5696.50 & 0.33 & 0.31 & 0.29 & 0.19 & 0.12 & 0.13 & 0.05 & 0.07 & 0.16 & 0.07 & 0.09 & 0.08 & 0.09 & 0.08 & 0.07 \\
\hline 5727.50 & 0.51 & 0.47 & 0.43 & 0.28 & 0.20 & 0.17 & 0.09 & 0.10 & 0.19 & 0.09 & 0.12 & 0.11 & 0.13 & 0.12 & 0.11 \\
\hline 5757.50 & 0.50 & 0.48 & 0.42 & 0.35 & 0.24 & 0.19 & 0.11 & 0.12 & 0.22 & 0.12 & 0.15 & 0.14 & 0.15 & 0.14 & 0.13 \\
\hline 5788.50 & 0.39 & 0.35 & 0.34 & 0.25 & 0.14 & 0.12 & 0.04 & 0.06 & 0.17 & 0.06 & 0.07 & 0.06 & 0.07 & 0.06 & 0.04 \\
\hline 5819.50 & 0.40 & 0.37 & 0.38 & 0.22 & 0.16 & 0.13 & 0.03 & 0.05 & 0.17 & 0.04 & 0.05 & 0.04 & 0.05 & 0.04 & 0.03 \\
\hline 5849.50 & 0.49 & 0.43 & 0.47 & 0.29 & 0.18 & 0.17 & 0.05 & 0.09 & 0.23 & 0.08 & 0.09 & 0.08 & 0.09 & 0.07 & 0.04 \\
\hline 5941.50 & 0.54 & 0.51 & 0.50 & 0.33 & 0.21 & 0.18 & 0.07 & 0.10 & 0.23 & 0.09 & 0.10 & 0.09 & 0.11 & 0.09 & 0.08 \\
\hline 5972.50 & 0.42 & 0.43 & 0.40 & 0.30 & 0.17 & 0.16 & 0.07 & 0.09 & 0.21 & 0.09 & 0.10 & 0.08 & 0.10 & 0.09 & 0.06 \\
\hline 6001.50 & 0.47 & 0.46 & 0.45 & 0.33 & 0.21 & 0.18 & 0.08 & 0.10 & 0.21 & 0.08 & 0.10 & 0.08 & 0.10 & 0.10 & 0.07 \\
\hline 6032.50 & 0.51 & 0.48 & 0.45 & 0.33 & 0.20 & 0.18 & 0.08 & 0.11 & 0.22 & 0.09 & 0.10 & 0.08 & 0.10 & 0.10 & 0.08 \\
\hline 6062.50 & 0.52 & 0.46 & 0.42 & 0.27 & 0.15 & 0.14 & 0.05 & 0.06 & 0.20 & 0.07 & 0.10 & 0.09 & 0.10 & 0.09 & 0.07 \\
\hline 6093.50 & 0.65 & 0.58 & 0.51 & 0.37 & 0.22 & 0.21 & 0.11 & 0.13 & 0.24 & 0.12 & 0.17 & 0.16 & 0.18 & 0.17 & 0.16 \\
\hline 6123.50 & 0.65 & 0.58 & 0.56 & 0.37 & 0.20 & 0.19 & 0.08 & 0.10 & 0.25 & 0.10 & 0.13 & 0.12 & 0.13 & 0.12 & 0.10 \\
\hline 6154.50 & 0.68 & 0.62 & 0.60 & 0.42 & 0.27 & 0.21 & 0.10 & 0.12 & 0.27 & 0.12 & 0.13 & 0.12 & 0.13 & 0.12 & 0.09 \\
\hline 6185.50 & 0.62 & 0.59 & 0.57 & 0.39 & 0.25 & 0.21 & 0.10 & 0.12 & 0.27 & 0.12 & 0.14 & 0.12 & 0.13 & 0.12 & 0.09 \\
\hline 6215.50 & 0.62 & 0.54 & 0.53 & 0.32 & 0.18 & 0.24 & 0.13 & 0.16 & 0.33 & 0.17 & 0.18 & 0.17 & 0.18 & 0.16 & 0.13 \\
\hline 6307.50 & 0.59 & 0.56 & 0.53 & 0.36 & 0.21 & 0.20 & 0.10 & 0.12 & 0.27 & 0.13 & 0.14 & 0.14 & 0.15 & 0.14 & 0.11 \\
\hline 6338.50 & 0.49 & 0.51 & 0.47 & 0.37 & 0.20 & 0.20 & 0.10 & 0.13 & 0.27 & 0.13 & 0.15 & 0.14 & 0.16 & 0.15 & 0.11 \\
\hline 6366.50 & 0.59 & 0.57 & 0.53 & 0.41 & 0.23 & 0.26 & 0.15 & 0.16 & 0.30 & 0.15 & 0.16 & 0.15 & 0.17 & 0.16 & 0.14 \\
\hline 6397.50 & 0.68 & 0.64 & 0.71 & 0.47 & 0.25 & 0.25 & 0.13 & 0.15 & 0.27 & 0.12 & 0.14 & 0.13 & 0.15 & 0.14 & 0.11 \\
\hline 6427.50 & 0.75 & 0.66 & 0.57 & 0.39 & 0.24 & 0.26 & 0.13 & 0.15 & 0.29 & 0.14 & 0.15 & 0.15 & 0.16 & 0.15 & 0.11 \\
\hline 6458.50 & 0.75 & 0.70 & 0.63 & 0.47 & 0.29 & 0.29 & 0.17 & 0.19 & 0.32 & 0.18 & 0.20 & 0.19 & 0.21 & 0.20 & 0.17 \\
\hline 6488.50 & 0.86 & 0.79 & 0.69 & 0.54 & 0.31 & 0.30 & 0.18 & 0.20 & 0.36 & 0.19 & 0.21 & 0.20 & 0.21 & 0.19 & 0.17 \\
\hline 6519.50 & 0.74 & 0.69 & 0.62 & 0.47 & 0.25 & 0.28 & 0.16 & 0.18 & 0.33 & 0.16 & 0.17 & 0.17 & 0.18 & 0.17 & 0.13 \\
\hline 6550.50 & 0.60 & 0.57 & 0.55 & 0.43 & 0.21 & 0.24 & 0.15 & 0.17 & 0.32 & 0.17 & 0.18 & 0.17 & 0.18 & 0.17 & 0.14 \\
\hline 6580.50 & 0.61 & 0.59 & 0.57 & 0.43 & 0.20 & 0.27 & 0.15 & 0.18 & 0.35 & 0.19 & 0.21 & 0.20 & 0.20 & 0.17 & 0.13 \\
\hline
\end{tabular}

Note. ${ }^{\text {a }}$ See the notes in Table 1.

via comparisons to numerous independent data sets. We consider the close agreement between these two sufficiently, to our reckoning, independent evaluations of the $\mathrm{Mg}$ II changes as a confirmation that any unaccounted instrumental biases do not significantly affect the new estimates in the UV1 spectral region.

To show in more detail the SSI changes during the current Cycle 24 and reveal subtle line-variability patterns, we return to the individual pair-wise differences (e.g., 2013 August-2008 August) and create two average spectra: the first group comprises the current solar maximum (i.e., all the data with $t>5900$ days), the second average covers the solar minimum $(4300<$ $t<5100$ days). Wavelength-binning of the normalized difference to $0.2 \mathrm{~nm}$ in UV1 and $0.05 \mathrm{~nm}$ in UV2 and VIS produces a long-term variability spectrum (Figure 3 ). We discuss the linevariability patterns in Section 3.2. Note that the abundance of data contributing to the long-term difference spectrum, when coupled with the "spectral smile" effect, allows us to decrease the original wavelength sampling step in the UV2 and VIS domains by $3-4 \times$ without any substantial increase of noise level, thus providing more detailed snapshot of the long-term lineprofile variability.

\subsection{The Maximum-Minimum Difference Spectrum from Rotational Modulation}

How do the long-term (years) SSI changes compare to the short-term (days-months) trends? We evaluate SSI changes for 27 day rotational modulation cycles by simply using the individual daily measurements, rather than applying the regression analysis proposed by Heath \& Schlesinger (1986). The excellent short-term (months) wavelength and throughput stability of the OMI instrument (see the estimates of degradation rates in
Appendix B) allows us to co-add the differences coming from relatively distant 27 day cycles. In doing so, we are helped by the fact that between summer 2012 and spring 2013 the Sun went through a series of pronounced rotational modulations (up to $4 \%$ peak-to-valley in the $\mathrm{Mg}$ II index), while approaching and going through the maximum of solar Cycle 24. We choose eight rotational cycles of roughly equal amplitude (shown in Figure 1 with the chosen maxima marked by vertical lines), create 16 max-min differences (between a maximum and two adjacent minima), normalize each difference and finally average the normalized differences, showing them in Figure 3 along with the long-term trends. Under this routine, a typical maximum or minimum spectrum comprises from 2 to 10 daily solar spectra, with the size of the time-averaging interval depending on the shape of the particular minimum or maximum. Besides, one ought to remember that each daily solar spectrum includes up to 60 individual (row-wise) spectra, with each measurement equally contributing to the average of up to $\sim 120-600$ spectra.

We also repeat this exercise for eight cycles from 2004 December through 2005 July, i.e., on a descending branch from the maximum of Cycle 23. This particular epoch provides relatively low, 1.8\%-3.2\% in the Mg II index values, 27 day modulation, and is used for a consistency check. These two (2005 and 2012) epochs show similar, to within $\sim 0.1 \%$, uncertainty limits (see below), variability patterns, after we account for the difference in the average modulation amplitude. The only adjustment required to reach an agreement between two observational epochs is the use of a single wavelength-independent multiplicative factor determined at the peak of the $\mathrm{Mg}$ II feature.

Comparison of the previously published normalized SSI changes from Cycle 21 (DeLand \& Cebula 1993) and the new OMI 27 day difference also shows a close, to within 

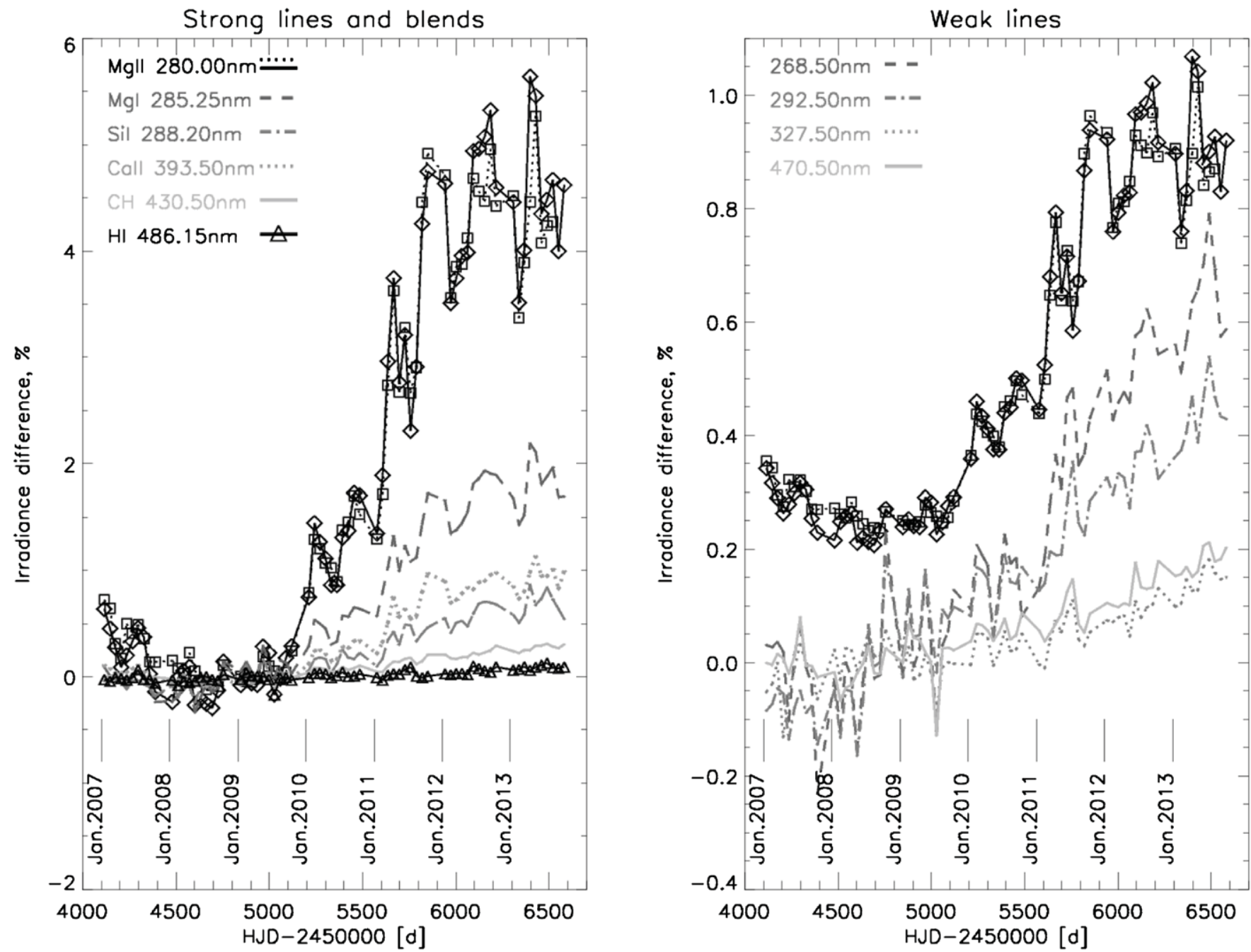

Figure 2. Time dependence of the long-term SSI changes for Cycle 24. The long-term differences are averaged within 0.8-5.0 nm intervals centered on specific wavelengths, which are listed in each panel. Squares in the left panel follow the normalized Mg II indices from DeLand \& Marchenko (2013), diamonds show the newly derived average solar flux differences for the wavelength bin centered on $\mathrm{Mg}$ II, and the triangles mark the $\mathrm{H} \beta$ measurements. For comparison with the "continuum" data, these Mg II data, scaled and shifted for clarity, are also plotted in the right-hand section. Note the difference in $y$-axis scales for the panels.

Table 3

The Normalized Max-Min Difference Spectra Derived from the 27 day Rotational Modulation

\begin{tabular}{lcc}
\hline \hline $\begin{array}{l}\text { Wavelength } \\
(\mathrm{nm})\end{array}$ & $\begin{array}{c}\text { Difference } \\
(\%)\end{array}$ & $\begin{array}{c}\text { Error } \\
(\%)\end{array}$ \\
\hline 170.0 & 1.308 & 0.078 \\
170.2 & 1.219 & 0.133 \\
170.4 & 1.462 & 0.123 \\
170.6 & 1.323 & 0.085 \\
170.8 & 1.222 & 0.094 \\
\hline
\end{tabular}

(This table is available in its entirety in a machine-readable form in the online journal. A portion is shown here for guidance regarding its form and content.)

uncertainties, agreement (Figure 4) after the new data are appropriately adjusted by matching the peak of the $\mathrm{Mg}$ II doublet. Encouraged by the remarkable repeatability of the results spanning more than two decades of satellite observations, we re-bin the new OMI short-term differences on a uniform $0.2 \mathrm{~nm}$ wavelength grid, appropriately adjust them (again, using the top of the $\mathrm{Mg}$ II profile) to the 27 day spectrum from DeLand \& Cebula (1993) and list the composite, normalized SSI changes in Table 3 . The large, FWHM $\sim 3 \mathrm{~nm}$ in the OMI spectra, width of the Mg II doublet justifies implementation of the top-of-the profile adjustment for the subsets of different $(0.6 \mathrm{~nm}$ versus $1.1 \mathrm{~nm}$ ) spectral resolution. We use the DeLand \& Cebula (1993) data, derived primarily from Cycle 21 measurements, in the $\lambda 170-265 \mathrm{~nm}$ region, and the OMI Cycle 24 measurements in the $\lambda 265-500 \mathrm{~nm}$ range. Recently, DeLand \& Cebula (2012) showed that their results derived from earlier measurements were also consistent with short-term SSI variations observed in multiple concurrent data sets during the declining phase of Cycle 23.

We have also examined the GOME- $2^{3}$ solar spectra, which provide additional spectral coverage out to $800 \mathrm{~nm}$. We precisely match the dates of the 2012-2013 OMI time sampling (eight maxima from Figure 1), create 16 max-min GOME-2 difference spectra, average the differences and plot the mean 27 day GOME-2 spectrum, without any further adjustments, in Figure 5. Though the $\sim 2 \times$ higher spectral GOME- 2 resolution results in more pronounced variability in the cores of prominent spectral lines and blends (e.g., the Fe I blends at $\lesssim \sim 300 \mathrm{~nm}$,

\footnotetext{
3 http://www.eumetsat.int/website/home/Satellites/CurrentSatellites/Metop/
} MetopDesign/GOME2/index.html 

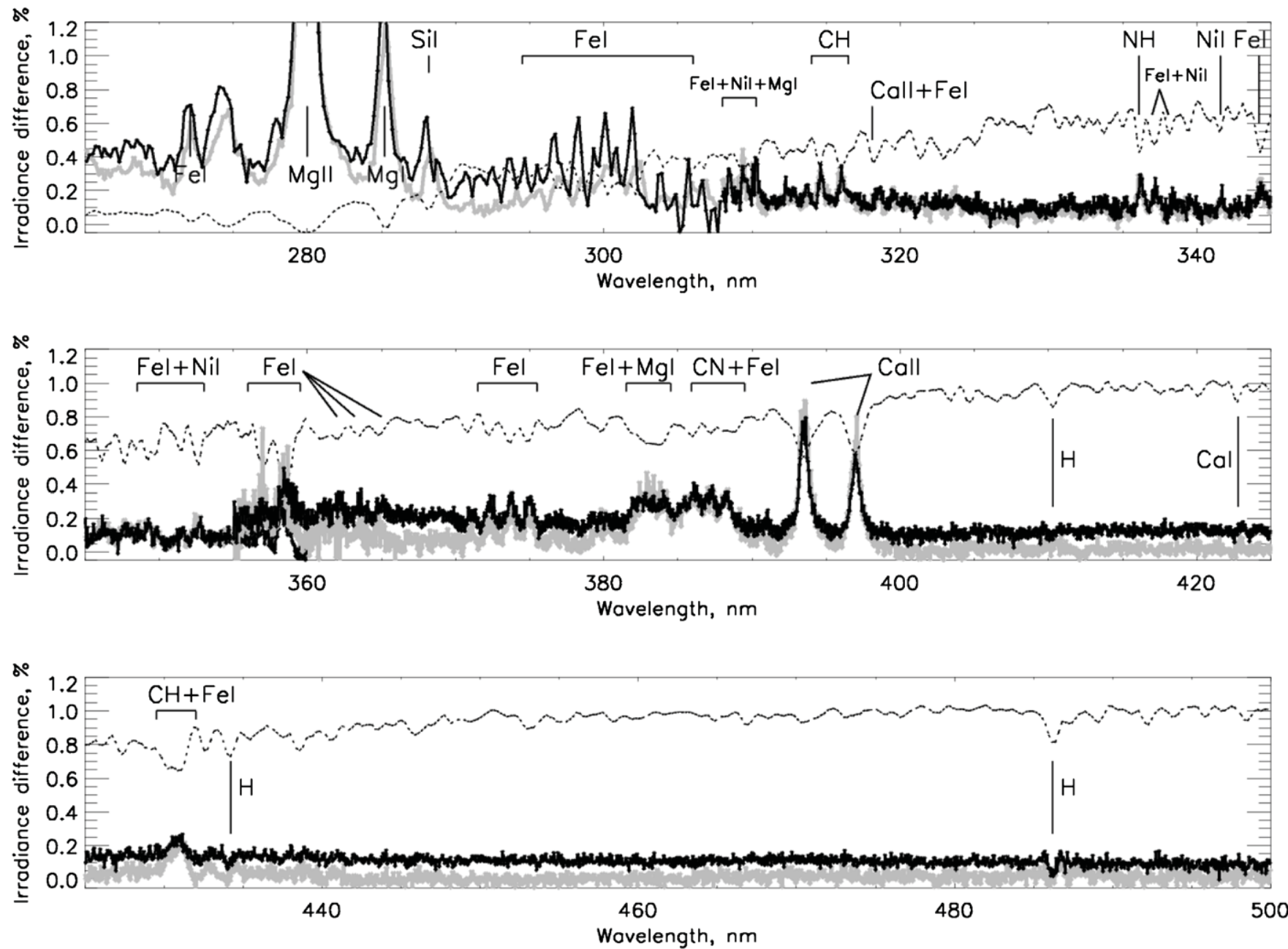

Figure 3. Spectral dependence of solar variations as observed by OMI. Black line shows the normalized long-term difference spectrum (2012-2013 vs. 2007-2009), properly adjusted, by using the wavelength-independent multiplicative factor, to match the top of the $\mathrm{Mg}$ II $280 \mathrm{~nm}$ profile from the average 27 day variability spectrum (gray line). For reference, the scaled solar spectrum is shown as a dotted line. The major solar lines and line blends are identified following Wallace et al. (2011).

Ca II lines), the OMI and GOME-2 27 day differences show essentially the same wavelength dependence, with the activity level gradually rising toward shorter wavelength. This agreement allows us to extend our observed solar short-term difference results toward longer wavelengths. We re-bin the GOME-2 differences to a uniform $0.2 \mathrm{~nm}$ wavelength step, re-scale them using the same scaling factor as in the adjustment of the OMI and DeLand \& Cebula (1993) data sets, and add the re-binned and re-scaled 500-800 nm GOME-2 data to the results listed in Table 3.

\subsection{Uncertainties in the SSI Differences}

We assign the overall $0.2 \%$ accuracy to the long-term SSI variations provided in Tables 1 and 2 (see Appendix B for more details). This assessment is influenced by a superposition of different sources of systematic errors. The most influential factor is the seasonal changes of the solar goniometry that lead to coherent variations over broad regions of the solar spectrum. Careful choice of the reference epochs, as well as epoch-dependent assessment of the long-term differences, bring the biases down to the $\sim 0.1 \%-0.2 \%$ level. The second major source of systematic errors stems from the proposed degradation model of the OMI throughput. These errors were minimized in the course of a multi-step, iterative regression procedure applied to the OMI 2007-2009 irradiances (see Appendix B).

As we already mentioned, an additional validation of the degradation model, though limited to the short wavelength region, is provided by the $\mathrm{Mg}$ II indices derived from the OMI data (DeLand \& Marchenko 2013). The indices passed multiple external checks, including the comparison with contemporary observations of the solar flux at $10.7 \mathrm{~cm}$. We noted the close match between the model-reliant measurements of the solar flux at $\lambda=280 \mathrm{~nm}$ and the fairly bias-free solar Mg II index (Figure 2). Indeed, these two relatively independent estimates are highly correlated, $r=0.994$, while the average difference between the $\mathrm{Mg}$ II line-flux and the $\mathrm{Mg}$ II index comes to $0.16 \% \pm 0.03 \%$, to be compared to the $\sim 4.5 \%$ solar Cycle 24 max-min amplitude (Figure 2, left panel).

The relatively small, $\lesssim 0.2 \%$, but systematic differences between the spectra coming from two independent approaches (the long-term and the 27 day difference spectra) highlight shortcomings of the applied degradation model in the long-term approach; e.g., one may note the distinctive $\sim 0.2 \%$ step between the UV2 and VIS channels seen in the long-term difference at 355-360 $\mathrm{nm}$ (Figure 3), contrasted by a smooth behavior of the 27 day difference in the same region. In addition, we suspect that the relatively high-amplitude pattern seen in the long-term 

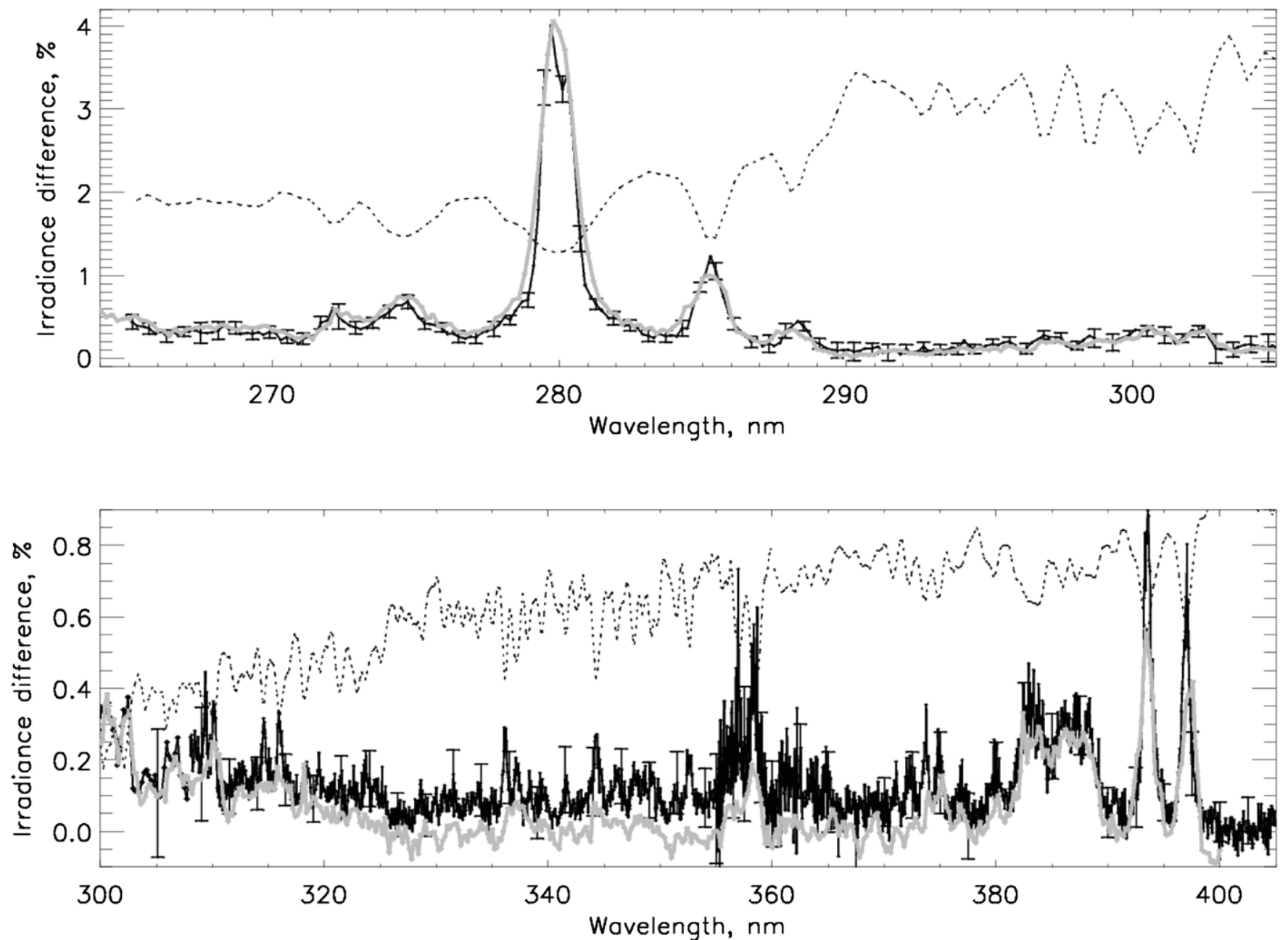

Figure 4. Black line: the normalized 27 day difference spectrum (an average of eight cycles between 2012 June and 2013 April), with representative $\pm 1 \sigma$ error bars. Gray line: the adjusted scaling factors from DeLand \& Cebula (1993). Dotted line: the scaled solar spectrum.

difference around the $290-310 \mathrm{~nm}$ region can be ascribed to an unaccounted gradual wavelength drift in UV1. Hence, we regard the 27 day spectrum (Table 3 ) as a better representation of the intrinsic SSI variability, with a noteworthy exception of few hydrogen lines (see Section 3.2). We retain the more prone to instrumental biases data from Tables 1 and 2 in order to better document the SSI changes in solar Cycle 24 and to reinforce the statement that, save some minor exceptions, the short-term rotational and long-term solar-cycle variability rates are consistent to within the quoted systematic errors.

The formal uncertainties for the 27 day difference, derived from the data grouped from different rotational cycles, and different rows (cross-track positions), within either $0.2 \mathrm{~nm}$ (UV1) or $0.05 \mathrm{~nm}$ (UV2 and VIS) wavelength bins, point to $\sigma \sim 0.05 \%-0.10 \%$ across the spectrum. Inspecting the average deviations between the individual differences for each rotational cycle, we assign the overall $0.1 \%$ uncertainty to the scale factors coming from the 27 day rotational modulation.

\section{DISCUSSION}

\subsection{The Short- and Long-term SSI Changes}

The results presented in Section 2.2 and Figure 4 show very good agreement between the 27 day SSI variations derived from OMI data and the DeLand \& Cebula (1993) scale factors, despite the limited signal-to-noise capability of the earlier-epoch scale-factor analysis at longer wavelengths. The only spectral region that shows any systematic differences is a short interval near $350 \mathrm{~nm}$, where the higher OMI spectral resolution may capture more Fraunhofer line-structures. There is also a good agreement between the Cycle 23 SSI changes reconstructed from the SCIAMACHY observations and the combined OMI and GOME-2 data: cf. our Figure 5 and Figure 13 from Pagaran et al. (2009). In particular, we note that the SSI changes remain consistently positive at $\lambda<800 \mathrm{~nm}$ in both (OMI+GOME-2 and SCIAMACHY) data sets, thus contrasting the SORCE/SIM and SORCE/SOLSTICE results. We understand that the transition from facular-dominated irradiance variations at UV wavelengths to sunspot-dominated irradiance variations at visible wavelengths limits the physical connection of these scaling factors to long-term irradiance changes. Nevertheless, we consider it important that the estimated variations derived from the scaling factors and $\mathrm{Mg}$ II index do in fact consistently represent even small long-term variations in solar irradiance at near-UV and visible wavelengths. Lean \& Woods (2012) note that a diskintegrated proxy such as the Mg II index is particularly useful for tracking TSI variations, because it avoids the difficulty of accurately identifying and quantifying all facular contributions needed as input to an image-based model calculation of irradiance variations. The agreement between scaling factors and 

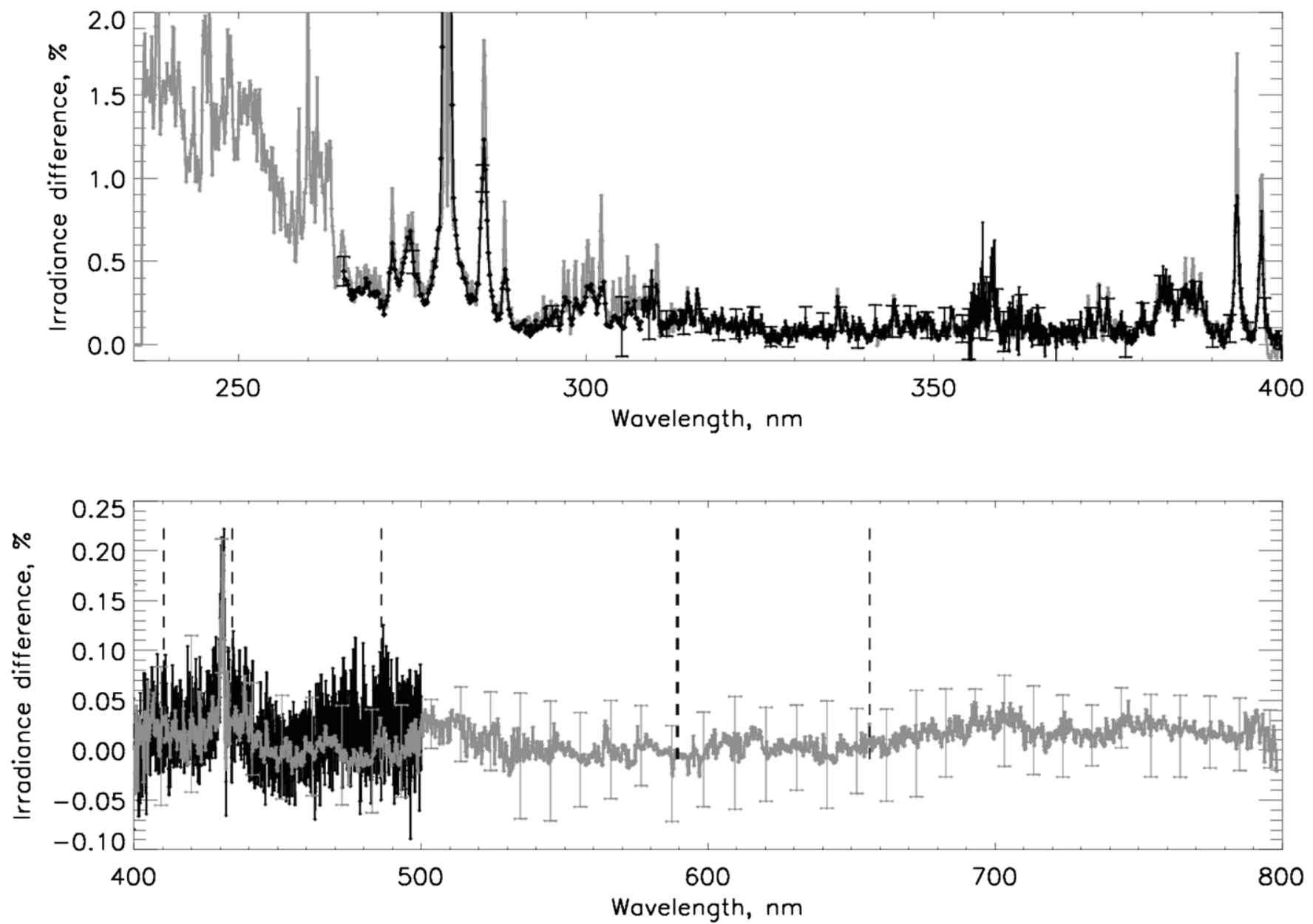

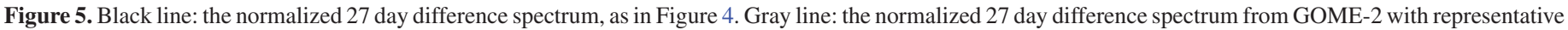
$\pm 1 \sigma$ error bars. Dashed lines mark the positions of hydrogen and Na I D lines.

observed long-term irradiance variations has motivated us to create the extended data set provided in Table 3 as a convenience for other users.

The magnitude of the discrepancy between SIM V17 longterm irradiance variations reported by Harder et al. (2009) and other results, such as variations predicted by NRLSSI (see Ermolli et al. 2013 and references therein), is particularly large at near-UV and visible wavelengths. As discussed by DeLand \& Cebula (2012), the SIM results imply solar-cycle changes of $\sim 3.5 \%$ at $290-320 \mathrm{~nm}$ for a max-min range such as that observed in Cycle 23, decreasing to $\sim 2 \%$ at $350 \mathrm{~nm}$. It should be noted that at 300-350 nm the reported uncertainty for SIM irradiance trends is only $0.1 \%-0.2 \%$ during 2004-2007, based on inter-detector comparisons. SIM Version 19 (V19) irradiance data have recently been released, with extended spectral coverage (down to $240 \mathrm{~nm}$ ) and temporal coverage (out to 2011 April) compared to the V17 product. $^{4}$ The new SIM data product now shows irradiance decreases as large as $-0.5 \%$ at wavelengths between 310 and $360 \mathrm{~nm}$ during the rise in activity from solar minimum to 2011 April. Although the current Cycle 24 is clearly weaker than Cycle 23, the OMI long-term irradiance variations shown in Figures 2 and 3 (+0.1\%-0.2\% increase between 300 and $350 \mathrm{~nm}$ ) do not support either set of SIM results. We note also that DeLand \& Cebula (2012) showed that scale factors derived from SIM short-term

\footnotetext{
4 See online notes at http://lasp.colorado.edu/home/sorce/data/ssi-data/.
}

irradiance data variations during 2004-2005 were consistent with scale factors derived from concurrent measurements by other satellite instruments. Thus, the accuracy of the longterm degradation correction for the SIM instrument remains a concern.

The characterization of the magnitude and spectral dependence of solar variability is particularly important for studies of the Earth's atmosphere, where both photodissociation and heating by solar radiation are significant. Ermolli et al. (2013) provide an extensive review of recent atmospheric model simulations using different solar variation scenarios. In one specific example, Swartz et al. (2012) used two-dimensional model simulations to show that the stratospheric ozone response to SSI variations is effectively a linear combination of ozone production at $\lambda<242 \mathrm{~nm}$ and ozone loss at $\lambda>242 \mathrm{~nm}$. For example, at wavelengths longer than $242 \mathrm{~nm}$, the model results in Swartz et al. (2012) show a maximum sensitivity of $-1 \%$ ozone change at $45-50 \mathrm{~km}$ for every $1 \%$ change in irradiance (cf. $\sim 0.6 \%-0.8 \%$ Cycle-24 amplitudes at $\lambda 260-270 \mathrm{~nm}$ from Table 2). Previous solar measurements at these wavelengths have reported longterm uncertainties of approximately $2 \%$ (see DeLand \& Cebula 2008 and references therein), so that the estimated uncertainties of OMI results (Section 2.3 and Appendix B) is a clear improvement in this regard. The reported uncertainty for SORCE SIM data is also small. However, the inferred wavelength dependence of the long-term SORCE SSI variability remains a concern (Swartz et al. 2012). 
Table 4

The Linear Pearson Correlation Coefficients

\begin{tabular}{|c|c|c|c|c|}
\hline Correlation & Mean & $\sigma$ & Minimum & Maximum \\
\hline $\mathrm{MgII}^{\mathrm{a}} / \mathrm{SL}^{\mathrm{b}}$ & 0.95 & 0.07 & 0.73 & 0.99 \\
\hline $\mathrm{Mg}$ II $/ \mathrm{WL}^{\mathrm{c}}$ & 0.88 & 0.05 & 0.80 & 0.96 \\
\hline$|\mathrm{B}|^{\mathrm{d}} / \mathrm{SL}$ & 0.72 & 0.06 & 0.56 & 0.79 \\
\hline |B|/WL & 0.64 & 0.06 & 0.56 & 0.75 \\
\hline Area $^{\mathrm{e}} / \mathrm{SL}$ & 0.82 & 0.09 & 0.56 & 0.91 \\
\hline Area/WL & 0.71 & 0.07 & 0.61 & 0.82 \\
\hline
\end{tabular}

Notes.

a The Mg II data from Table 1.

b The "strong-line" data from Table 1.

c The "weak-line" data from Table 2.

$\mathrm{d}$ The magnetic field magnitude average.

e The sunspot area.

\subsection{The Line-profile Variability}

Inspecting the variability patterns in Figures 2 and 3, we find that, besides the pronounced strengthening of chromospherically sensitive lines ( $\mathrm{Mg}$ I, Mg II, Ca II), the changes are mainly driven by either the blends of strong Fe I lines or by the diatomic molecular radicals, with a minor contribution from $\mathrm{Ni}$ I, $\mathrm{Mg}$ I, and Ti I. There is a clear trend: the lines become progressively shallower with rising solar activity. The similar line-filling effect was observed by Mitchell \& Livingston (1991) in Cycle 21. However, we ought to point to the disparate trends observed during the minimum between Cycles 22 and 23 (Livingston et al. 2007). The variability in the strong $\mathrm{CH}, \mathrm{CN}$, and $\mathrm{NH}$ bands frequently exceeds the amplitudes in the adjacent $\mathrm{Fe}$ I blends of comparable optical depth. The $\mathrm{CN}$ band head at $388.3 \mathrm{~nm}$ is known to be highly sensitive to activity in the low chromosphere (Livingston et al. 2007).

The increase of Fe I excitation energy with increasing wavelength (e.g., $\sim 1 \mathrm{eV}$ for strong Fe I transitions around $300 \mathrm{~nm}$ versus $\sim 3-4 \mathrm{eV}$ at $\lambda \sim 500 \mathrm{~nm}$ ) could cause the disappearance (in fact, a decrease of the variability amplitudes below the $\sim 0.1 \%$ detectability threshold) of the line-filling effect in the max-min spectra at $\lambda>450 \mathrm{~nm}$ (Figure 3 ). The Fe I lines of progressively higher excitation energies form deeper in the photosphere (Grossmann-Doerth 1994), thus demonstrating less and less of the line-filling effect which is related to the level of chromospheric activity (Livingston et al. 2007). The change of the line-profile variability with wavelength follows a similar trend observed in the relatively line-free regions, where the solar flux comes from progressively higher regions in the photosphere, as one moves to shorter wavelengths (Fröhlich \& Lean 2004). Besides, the gradual decrease of the facula contrast toward longer wavelength (Ermolli et al. 2007) also contributes to (if not dominates in) the observed decrease of the line-filling effect.

Using the wavelength-averaged data from Tables 1 and 2, we find very high degrees of correlation between the variations in all listed strong spectral features and the Mg II line: the linear Pearson correlation coefficients run between 0.93 and 0.99 , with the sole exception of $\mathrm{H} \beta$ with $r=0.73$. In Table 4, we provide the average correlation coefficients and corresponding standard deviations, as well as the min-max values of the coefficients. A high degree of correlation, $\sim 0.90-0.97$, between the $\mathrm{Mg}$ II index and the regression fits to the wavelength-binned solar irradiances from two spectral regions, $185-215 \mathrm{~nm}$ and $230-262 \mathrm{~nm}$, was also noted in the corrected Nimbus-7 data by DeLand \& Cebula (2001). In our data, the changes in $\mathrm{Mg}$ II are also correlated to the changes in the solar "continuum" (designated as "WL" in Table 4). However, there we note some wavelength dependence: the continuum is strongly correlated to the $\mathrm{Mg}$ II line flux at $\lambda=265-310 \mathrm{~nm}(r=0.90-0.96)$, but the correlation drops to $r=0.74-0.88$ for $\lambda>320 \mathrm{~nm}$. A gradual (though much more evident toward longer wavelengths) decline of correlation between the detrended UARS SOLSTICE SSI and the detrended $\mathrm{Mg}$ II facular proxy prompted introduction of the sunspot proxies into the NRLSSI model (Lean et al. 1997).

Earlier we reported a strong correlation between the $\mathrm{Mg}$ II index and F10.7 flux (DeLand \& Marchenko 2013). Now we expand this conclusion on the average magnitudes of the solar magnetic field and the average sunspot areas, correlating them with the strong-line (SL) and weak-line (WL) measurements from Tables 1 and 2. We use the daily OMNI $2^{5}$ (King \& Papitashvili 2005) magnetic field estimates and the daily average sunspot areas provided by NOAO ${ }^{6}$ We further group the daily readings into monthly means centered on the dates from Tables 1 and 2 . The $\mathrm{H} \beta$ line consistently scores the lowest among the strong spectral features in all kinds of correlations. The strong relationship of the line-flux changes (note that the $\mathrm{Mg}$ II index is widely used as a facular proxy) to the sunspot areas suggests that the detected SSI variability could be reproduced by the NRLSSI model (Lean et al. 1997). Indeed, multiple comparisons of the new OMI observations taken at different phases of Cycle 24 closely, to within the instrumental errors, agree with the corresponding model forecasts (J. Lean, private communication, 2013, 2014).

The relatively low OMI spectral resolution prevents us from detecting line-profile variability for $\lambda>450 \mathrm{~nm}$, except the peculiar case of $\mathrm{H} \beta$ (see below). However, the groundbased data of much higher spectral resolution acquired in the 500-560 nm region show the anticipated SSI changes, as well as the expected gradual decline toward longer wavelengths of their amplitudes (Mitchell \& Livingston 1991). In general terms, we support the conclusion of White et al. (1987): in the solar activity cycle all relatively strong spectral features formed by neutral-metal blends (mostly by Fe I and Ni I), closely follow the changes in $\mathrm{Mg}$ II and $\mathrm{Ca}$ II $\mathrm{H}$ and $\mathrm{K}$ lines, albeit with different relative amplitudes. Here we extend this conclusion on the 27 day SSI changes.

Figure 3 demonstrates a good overall correspondence of the short-term and long-term variability patterns, save the localized regions with known instrumental problems. Moreover, the newly derived SSI changes closely follow, to within $0.1 \%$ errors, the appropriately adjusted scaling factors from DeLand \& Cebula (1993; Figure 4), as well as the 27 day difference spectra from GOME-2 (Figure 5). The Cycle 21 and Cycle 24 SSI changes come remarkably close, considering the differences in spectral resolution, the $>20 \mathrm{yr}$ time span between observations, and, especially, the different methodology of variability assessment. Such congruency stresses the universality of the linear-scaling approach (see DeLand \& Cebula 2012 for further discussion).

However, there are some subtle deviations from this seemingly universal principle of scalability. After a careful alignment of the tops of the Mg II variability profiles, we find that the long-term changes are less pronounced when compared to the 27 day difference spectra: while insignificant for $\lambda \lesssim$ $350 \mathrm{~nm}$, the difference tends to grow toward longer wavelengths (Figure 6). Overall, this rather subtle effect either barely exceeds

\footnotetext{
5 ftp://spdf.gsfc.nasa.gov/pub/data/omni/low_res_omni/

6 http://www.swpc.noaa.gov/ftpmenu/indices/old_indices.html
} 

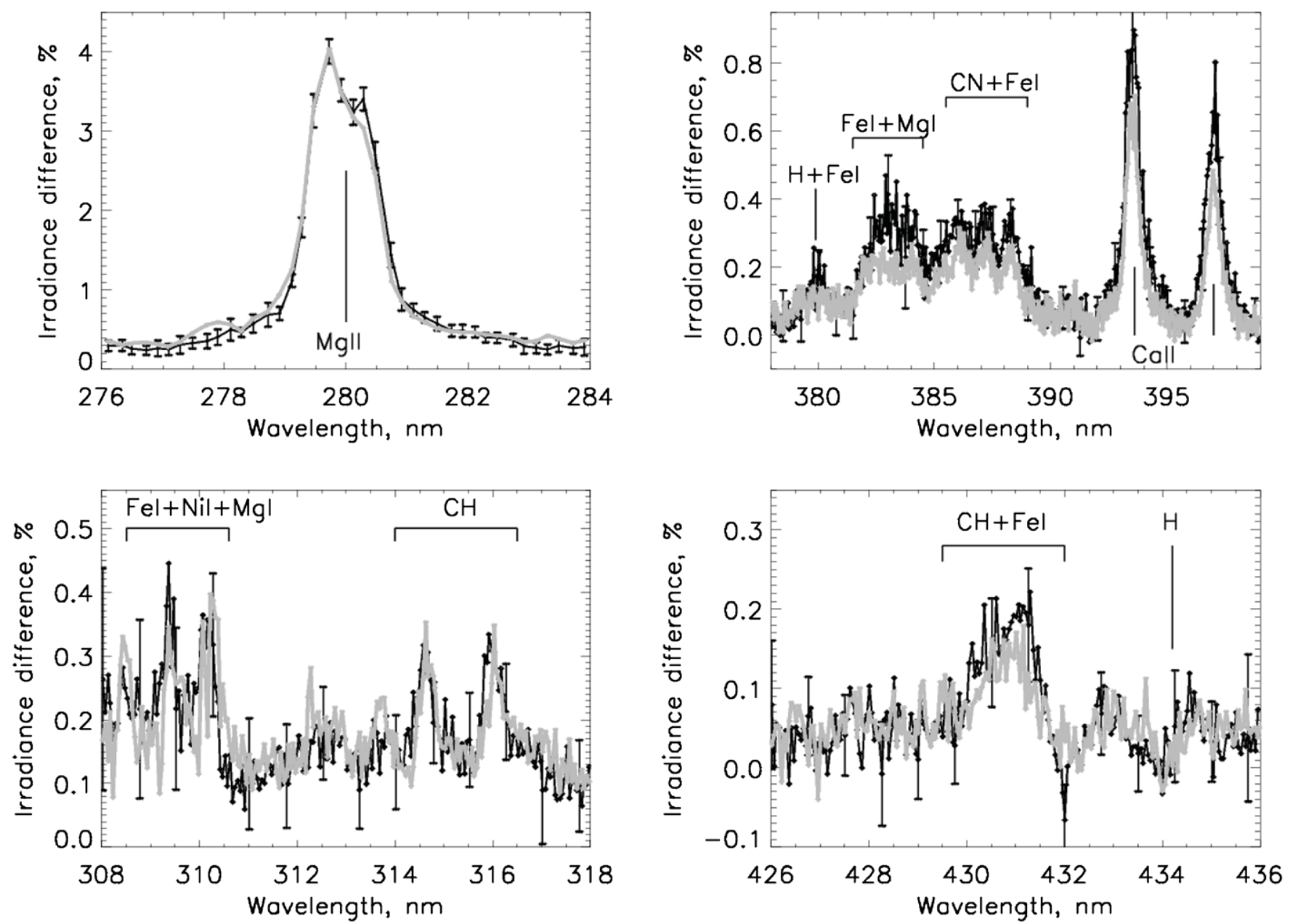

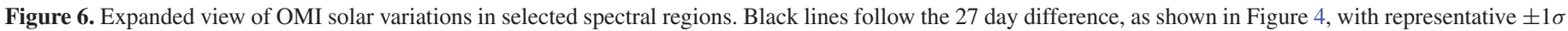

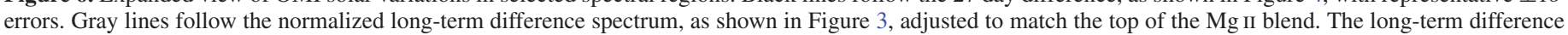
spectrum is shifted by $-0.03 \%$ in the upper left panel and by $-0.09 \%$ in the right-hand panels.

or stays well within the quoted observational uncertainties in this particular OMI data set. However, credibility of the observed trend is augmented by the results from Chandra et al. (1995; see also Woods et al. 2000): solar Cycle 22 also shows prominent differences between the 27 day and long-term SSI changes in the $\lambda \lesssim 170$ domain. Chandra et al. (1995) finds that the 27 day and long-term scale factors are similar in the 200-250nm range, while the long-term SSI changes are far more apparent compared to the rotational modulation in the $\operatorname{Ly} \alpha$ vicinity. Hence, combining the present findings with the results from Chandra et al. (1995), we see an apparent reversal of the trend: the long-term modulation prevails in the $\lambda \lesssim 170 \mathrm{~nm}$ range; the short- and long-term SSI changes have comparable, to within systematic errors, amplitudes in the $200-350 \mathrm{~nm}$ region; the 27 day variability gradually exceeds the long-term levels at $\lambda>350 \mathrm{~nm}$. As such, the finding calls for an independent confirmation and ensuing detailed interpretation. Here we mention that tentative explanations of the different wavelength dependence in the long-term and 27 day SSI variability may include wavelengthdependent contrasts between the active and quiet-Sun regions, the changing with wavelength integral output from active regions (e.g., the dominance of faculae at shorter wavelengths, then sunspots at longer wavelengths), as well as global solarcycle-related changes in the solar photosphere. However, the latter is still a debatable issue (Penza et al. 2006 and references therein).
The different reaction of lines on the 27 day and long-term perturbations extends to the observed hydrogen lines: $\mathrm{cf}$. the left and right panels in Figure 7. The upper Balmer lines, namely $\mathrm{H} \beta$ and $\mathrm{H} \gamma$, do not follow the general line-filling long-term trends (cf. the $\mathrm{CH}+\mathrm{Fe}$ I blend at $\lambda \sim 431 \mathrm{~nm}$ and $\mathrm{H} \gamma$ in the middle left-hand panel of Figure 7). Close inspection of Figure 2 and comparison of the $\mathrm{H} \beta$ data from Table 1 to the measurements of the adjacent continuum from Table 2 shows negligible, if not slightly "negative," relative line-filling effect for this particular line. To demonstrate the reality of this slight deviation, we turn to direct estimates of spectral line depths, measuring them in relevance to the adjacent relatively line-free regions. We normalize the data by the mean values around the solar minimum (Figure 1, HJD times = 4300-5100), produce three month linedepth averages, and plot time-series of these values in Figure 8. The prominent $\mathrm{Mg}, \mathrm{Ca}$, and $\mathrm{Fe}$ lines follow the expected trend: the high 2011-2013 solar activity results in shallower spectral lines. The markedly different reaction of hydrogen lines (getting slightly deeper at higher solar activity levels) could be related to the prevalence of the non-LTE effects in the upper Balmer and Paschen lines (Przybilla \& Butler 2004). This leads to profiles with relatively deeper line-cores at the epochs of heightened solar activity. Moreover, since the individual lines in the Balmer series form in different, though vertically (radially) overlapping, regions of the photosphere (Przybilla \& Butler 2004), the lines with progressively higher numbers end up being 

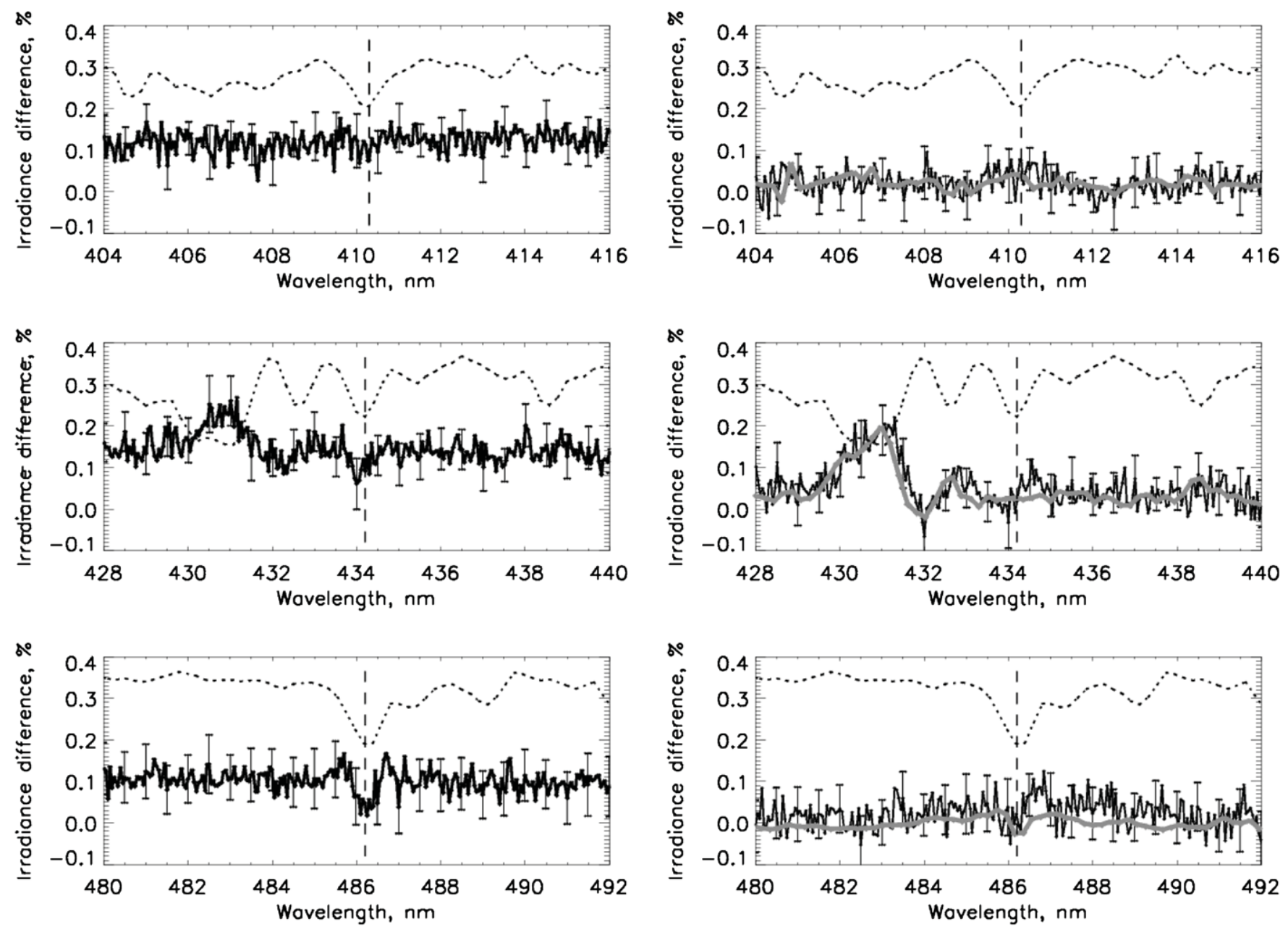

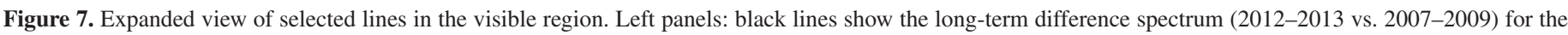

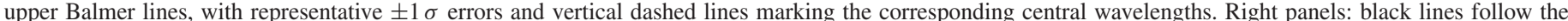
27 day OMI difference spectrum; gray lines: the similar 27 day GOME-2 spectrum. In all panels, the dotted lines show the scaled solar spectrum.

less susceptible to the non-LTE effects, hence showing less prominent deepening (cf. $\mathrm{H} \beta$ to $\mathrm{H} \delta$ in the left panel of Figure 7). Surprisingly, this core-deepening effect is seemingly inverted in the $\mathrm{H} \beta$ wings. Though superficially reminiscent of the doublepeaked appearance of the line-core in the quiet-Sun Ly $\alpha$ profile (Fontenla et al. 1988), the separation between the $\mathrm{H} \beta$ peaks far exceeds the $0.04 \mathrm{~nm}$ difference in the $\mathrm{Ly} \alpha$ case. The wings of strong absorption lines should, in general, form deeper in the photosphere; e.g., Fe I lines treated under the LTE approach (Grossmann-Doerth 1994). Considering the complex interplay between the changing opacities and temperature gradients (e.g., Fontenla et al. 2011), we abstain from any straightforward interpretation of this wing-reversal phenomenon, noting that the extent of the $\mathrm{H} \beta$ line-formation zone may lead to a situation where the line wings and the line core are governed by a timedependent (i.e., cycle-dependent) balance of excitation agents.

\subsection{A Brief Cautionary Note}

The spectral-fitting algorithms that retrieve trace-gas components in the Earth's atmosphere from the $\lambda \lesssim 400 \mathrm{~nm}$ domain (e.g., Coldewey-Egbers et al. 2005) and use a time-invariant (single-epoch) solar reference spectrum might be affected by the solar-line variability demonstrated by the OMI data. The problem could be substantially reduced, if not altogether avoided, by using the available Mg II index compiled since 1978 (see DeLand \& Marchenko 2013 and references therein) as a general scaling factor, then introducing its wavelength dependence (Table 3) and, finally, appropriately adjusting the single-epoch solar reference spectrum to the epoch of observations.

The consequences for planet searches around solar-like stars are as follows. The approaches based on broadband photometry should make an allowance for the line-profile variability induced by rotational modulation (e.g., Figure 5) on relatively short timescales, along with the customary reference to the putative long-term changes. Considering the gradual diminishing of the SSI changes with wavelength, such photometric variability could mainly affect the $\lambda<400 \mathrm{~nm}$ range, with a rapid growth toward shorter wavelength. Further quantitative estimates can be done using the normalized SSI variations from Table 3 (we remind the reader that these are derived from the 27 day rotational modulation), as a representative, to some extent, pattern of Sun-like variability on timescales from days to years, with relatively minor reservations regarding the behavior of hydrogen lines. Unfortunately, neither the Na I doublet nor the $\mathrm{H} \alpha$ line are observed by OMI, hence we cannot show the anticipated long-term (years) changes. These lines, along with the Ca II doublet, are frequently used as proxies in investigations of activity cycles in the late-type stars (e.g., Santos et al. 2010; Gomes da Silva et al. 2012; Robertson et al. 2013 and references 


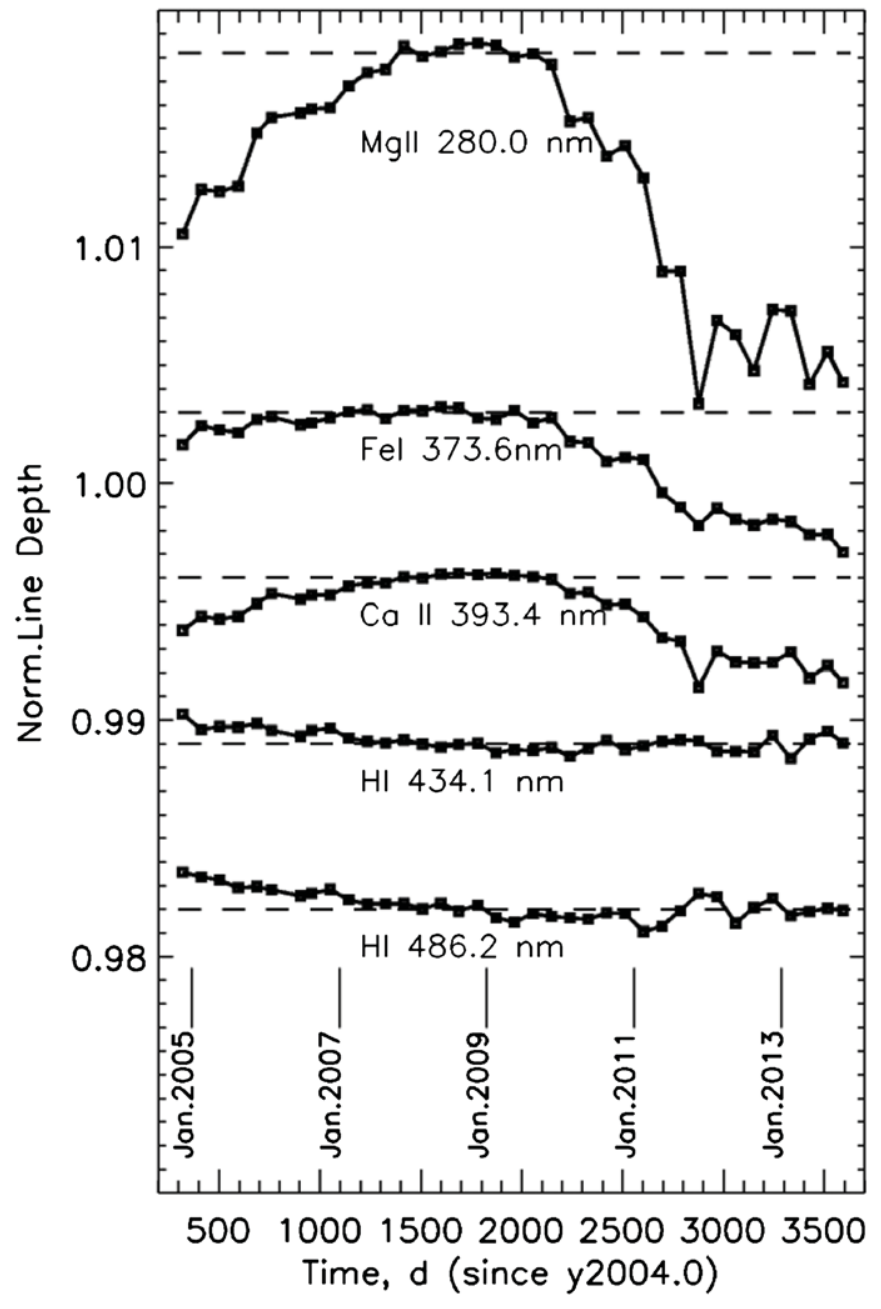

Figure 8. Normalized line depths of prominent absorption lines and blends. Dashed lines show the corresponding reference levels for each line, with arbitrary vertical shifts applied for clarity.

therein). One should note that the Sun shows good correlation between the $\mathrm{Ca}$ II and $\mathrm{H} \alpha$ activity indicators (Livingston et al. 2007; Meunier \& Delfosse 2009), while the late-type stars demonstrate bewilderingly complex trends (cf. Cincunegui et al. 2007; Santos et al. 2010). The confusion is exacerbated by the complete lack of detectable $(>0.05 \%)$ reaction of $\mathrm{H} \alpha$ and $\mathrm{Na}$ I $\mathrm{D}$ to the 27 day rotational modulation in the solar GOME-2 data, despite the clear involvement of Ca II (Figure 5).

\section{CONCLUSIONS}

Both the long-term (years) and short-term (weeks) SSI changes observed in OMI solar irradiance measurements during Cycle 24 closely match the relative amplitudes and wavelength dependence in the SSI variations derived from the solar Cycle 21 composite data (DeLand \& Cebula 1993), thus reinforcing the conclusion (DeLand \& Cebula 2012) about scalability of the solar-cycle (either short or long term) changes to variability of the $\mathrm{Mg}$ II index. We observe a general trend which shows that strong spectral lines and line blends become progressively shallower with increasing level of solar activity. The amplitude of changes gradually diminishes toward longer wavelengths. However, there are few exceptions. First, the upper Balmer lines defy this line-filling trend. Second, we noted the relatively small ( $\lesssim 0.1 \%$ under OMI spectral resolution) but seemingly systematic differences in variability amplitudes of prominent spectral blends and bands. Namely, the long-term (years) SSI changes seem to be less prominent compared to the changes induced by the 27 day rotation in the $\lambda \gtrsim 350 \mathrm{~nm}$ region. Even more surprisingly, the prominent hydrogen lines, as well as the Na I D doublet, do not show any changes related to a rotational cycle, despite the reported involvement in the longterm solar-cycle SSI changes along with the rest of metal and molecular lines. These remarkable "exceptions" call for an independent confirmation and, if proved to be real, clearly deserve further attention.

We are grateful to J. Joiner for suggestions helping to improve the content of the manuscript. The OMNI data were obtained from the GSFC/SPDF OMNIWeb interface at http://omniweb.gsfc.nasa.gov. This work was supported by the NASA grant \#NNG12HP08C.

\section{APPENDIX A}

\section{INSTRUMENT STABILITY}

Comprehensive monitoring of the OMI instrumental changes via different approaches (e.g., Dobber et al. 2008; Jaross \& Warner 2008; our own monitoring tools) shows good temporal stability of the instrument. As an example, we show the normalized spectral radiances observed over the low-reflectance geographical regions in UV1 and UV2 channels (Figure 9). The gradual changes in instrument sensitivity over seven years do not exceed $\sim 2 \%-3 \%$ in UV1 and UV2. However, since 2007 some of the Earth-observing fields-of-view (FOVs, i.e., rows) have been affected by the row anomaly. ${ }^{7}$ The currently prevailing interpretation of this condition links the anomalous spectral response to a combination of geometric blocking and out-of-FOV scattering of the solar light and Earth shine. The anomalous blocking and scattering is presumably caused by a warped thermal insulation partially obscuring some FOVs. The Sun-observing port is not directly involved in the row anomaly. However, since most of the optical elements are shared by the Earth- and Sun-observing channels (Dobber et al. 2006), the heightened rates of optical degradation are also registered in some UV1 FOVs. We do not account for these UV1 rows in the degradation model discussed in Appendix B, thus do not include the respective UV1 solar spectra in the reported max-min differences. Aside from the well-documented and comprehensively monitored changes in the areas affected by the row anomaly, the excellent temporal stability of the Earth-viewing pathway is closely matched by the small-scale and regular, thus quite predictable, instrumental changes in the Sun-observing channel (see below). To demonstrate this, we measure the central wavelength positions (defined as lineprofile centroids) of prominent spectral features in all three Sunviewing spectral channels, and plot the wavelength changes in Figure 10. In this figure, the Mg II doublet represents UV1, the Fe I $344 \mathrm{~nm}$ blend comes from UV2 and the strong Ca II line shows the remarkable long-term stability of the VIS channel. Overall, the instrumental drifts do not exceed $\sim 0.01 \mathrm{~nm}$ over the mission duration; this is to be regarded from a standpoint that OMI provides relatively low-resolution, $\delta \lambda=0.4-0.6 \mathrm{~nm}$, spectra. We note, however, that the detected wavelength shifts in the long-wavelength, $\lambda>290 \mathrm{~nm}$, UV1 range (not shown here)

\footnotetext{
7 See http://www.knmi.nl/omi/research/calibration/instrument_status_v3/ index.html.
} 

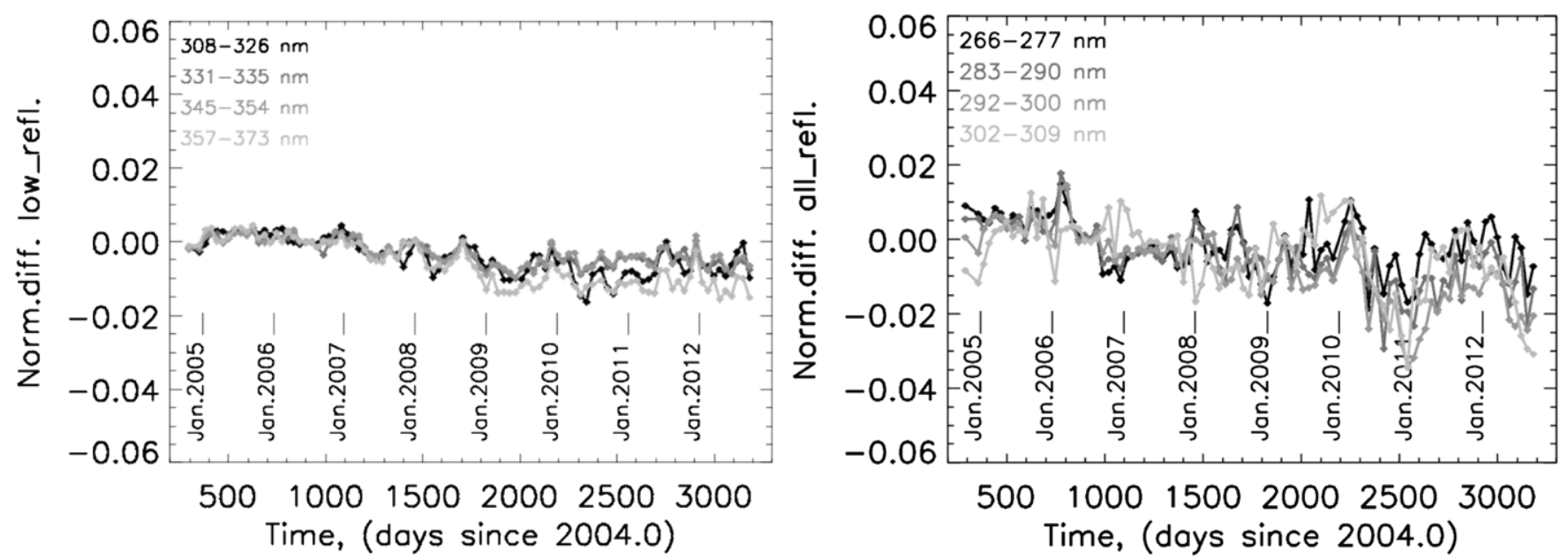

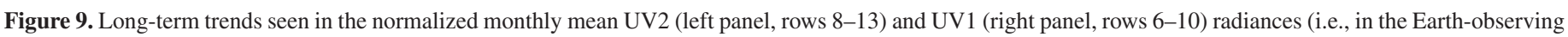
pathway). Different shades of gray correspond to the wavelength intervals shown in the upper-left corners.

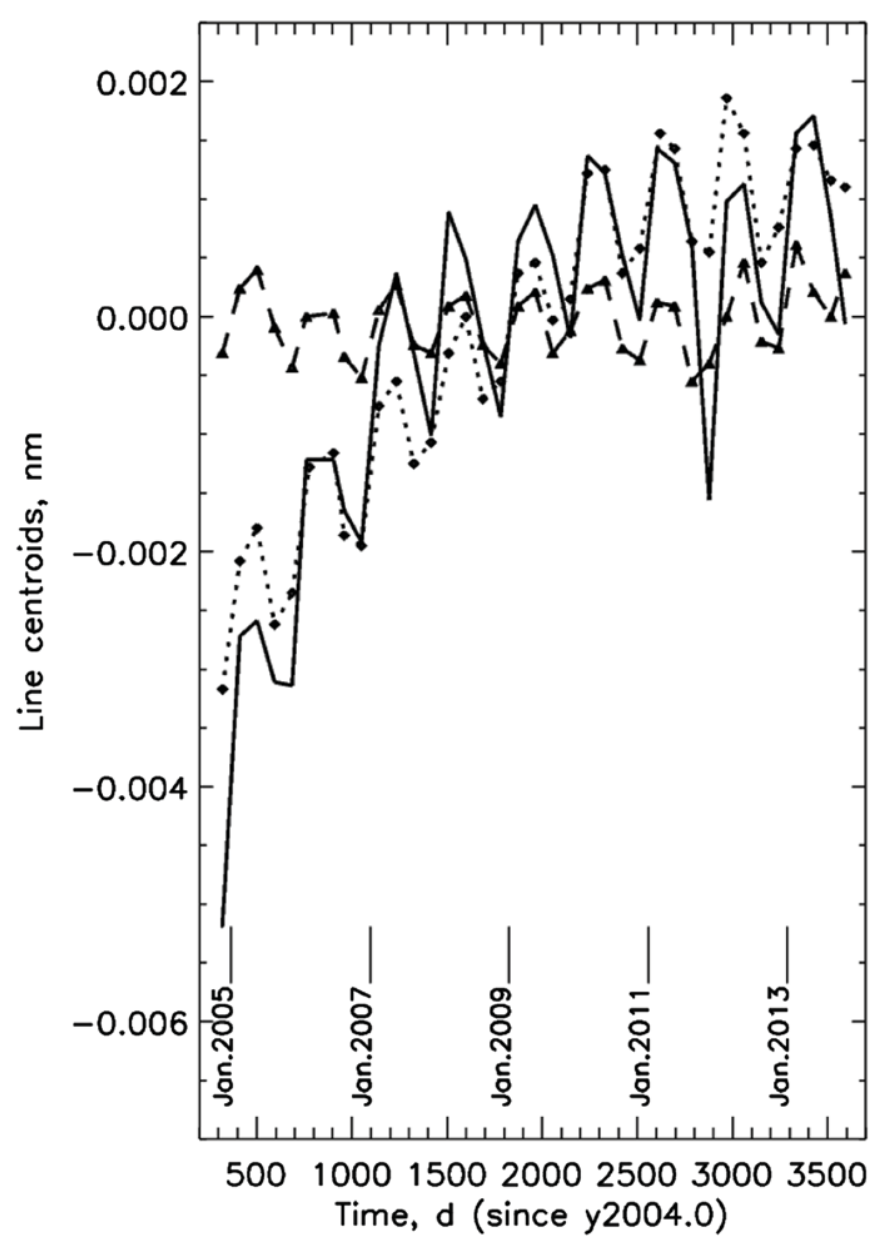

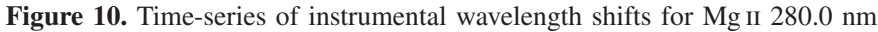
line (full line, UV1 channel), Fe I $344.2 \mathrm{~nm}$ blend (dotted line, UV2 channel), and Ca II $396.9 \mathrm{~nm}$ line (dashed line, VIS channel).

are more than twice as large as the changes observed around $280 \mathrm{~nm}$. These shifts might be responsible for the instrumental artifacts seen in the long-term maximum-minimum difference (Figure 3) at $\lambda>290 \mathrm{~nm}$.

Considering the small amplitudes of the reported SSI changes in the solar spectrum, any gradual stray light changes, which are not adequately captured by the currently implemented straylight compensation algorithm, may substantially bias the results. Comprehensive modeling shows that stray light contributes up to $\sim 40 \%$ to the Earth-shine spectrum in the $\mathrm{Mg}$ II line. Fortunately, the impact of this contribution dramatically lessens at longer wavelengths, falling well below $3 \%$ in the UV2 and VIS spectral lines. It can be shown that the majority of stray light is adequately captured by the currently implemented dataprocessing algorithm. The direct measurements of spectral line depths in the Earth-viewing channel show a long-term trend which, to within the yearly scatter, follow the line-shallowing trends in the solar data (cf. Figures 11 and 8), despite different stray-light contribution in the solar and Earth-viewing channels. In Figure 11, we plot three month averages of the solar line depths and individual daily data from the Earth-viewing channel, in order to highlight the influence of geophysical factors (Ring effect) on the latter. The Earth-view spectra were selected over low-reflectivity terrain without noticeable presence of absorbing aerosols. Moreover, the similarity of the $\mathrm{Mg}$ II variability patterns derived via different approaches (see Figure 2) vouches against any major change in the stray-light conditions in the Sun-observing pathway between 2007 and 2013. We remind the reader that this particular spectral line is highly sensitive to any instrumental biases introduced by putative changes in the stray-light level.

\section{APPENDIX B}

\section{THE SPECTRAL DEGRADATION MODEL}

In order to obtain the maximum-minimum difference spectrum from OMI solar measurements spread over years of observations, we should provide the estimates of changes in the instrument throughput with $\ll 0.5 \%$ accuracy. The OMI instrument is not equipped with direct, sufficiently accurate on board monitoring of such instrumental changes. However, we can take advantage of two factors to determine a long-term instrument characterization under the specific circumstances. First, there is ample evidence of the excellent instrumental stability (see Appendix A) gathered via indirect means. Second, the recent unusually protracted (Figure 1) solar minimum allows us to compile multi-year data records and, for this particular epoch (2007-2009), interpret all observed changes in the solar irradiances as of a pure instrumental origin. We then 

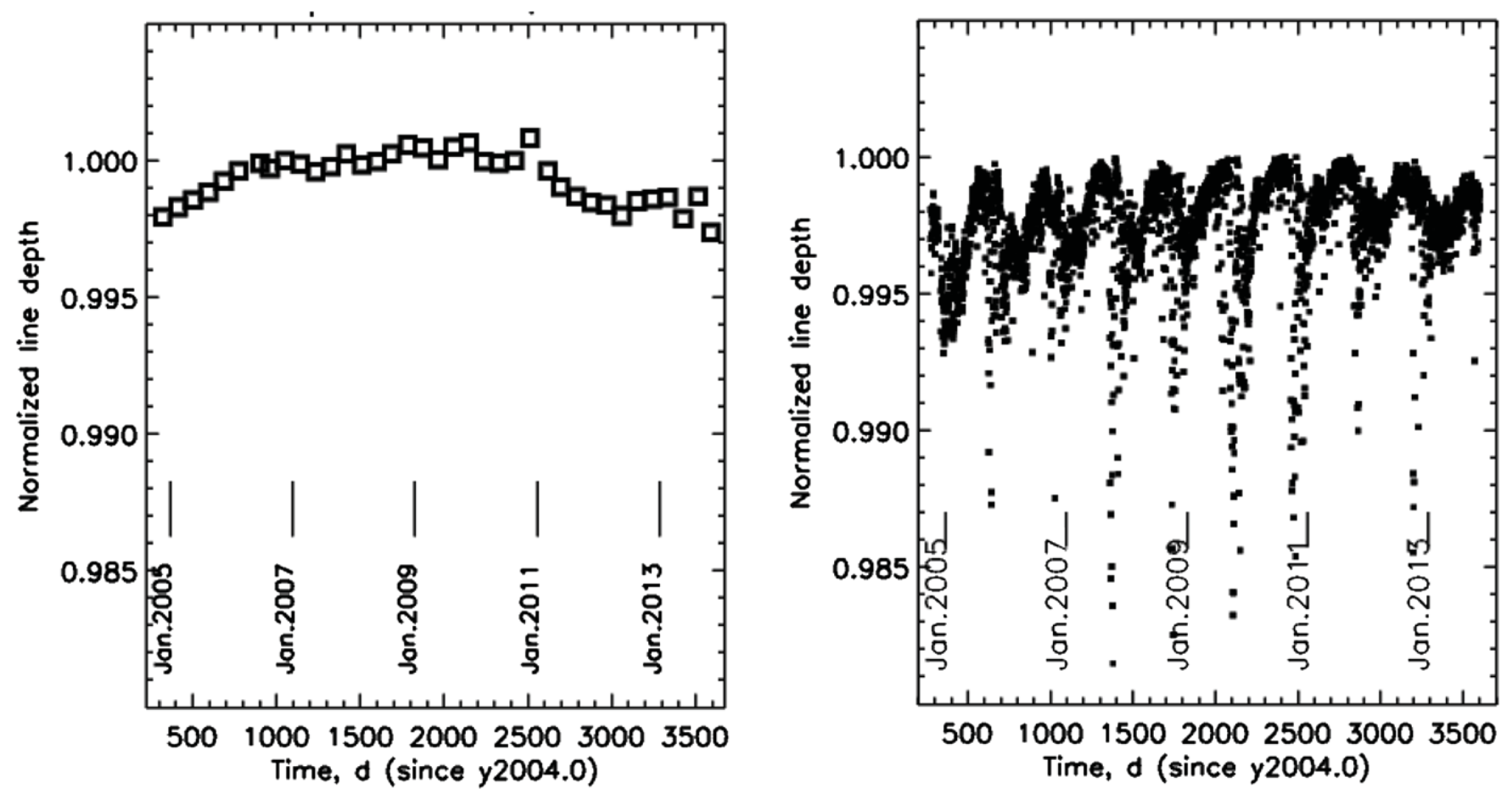

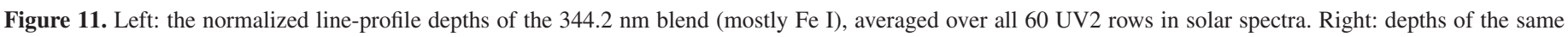
spectral blend in the Earth-view data, an average over rows 1-23, not affected by the row anomaly.

apply this characterization forward to determine instrument response changes for OMI solar irradiance measurements during Cycle 24 in 2009-2013. We implement the line-of-sight-, time-, and wavelength-dependent spectral degradation model for the Sun-observing optical pathways via a multi-step procedure.

1. Time-averaging of the daily solar observations, producing monthly averages of irradiances in three channels (UV1, UV2, VIS).

2. Wavelength binning; the monthly mean solar fluxes are further averaged at carefully selected relatively line-free, $2 \mathrm{~nm}$ wide regions (11 for UV1, 12 for UV2, and 20 for VIS); the degradation coefficients derived in steps 3 and 4 are calculated using these time- and wavelength-binned data.

3. The wavelength-dependent and line-of-sight-dependent (i.e., from an individual row) degradation rates are derived based on the data obtained during the solar 2007-2009 minimum (Figure 1). We exclude the periods of relatively unstable solar measurements caused by rapidly changing solar goniometry, which are clustered around November-January epochs, as shown in Figure 12.

4. The row (i.e., line of sight) and wavelength dependencies of the degradation coefficients from step 3 are approximated by linear functions, treating each spectral channel separately (Figure 13).

5. The sets of degradation coefficients calculated in step 4 provide forward-extrapolated (from 2007 onward) degradation rates for the monthly averages from step 1; each spectral channel is treated separately.

To our knowledge, most satellite instruments tend to show approximately linear degradation by the start of our analysis period (i.e., two to three years after launch). The fact that the beginning portion of the uncorrected time series in Figure 12 is below the extrapolated fit line, whereas the addition of timedependent solar activity would be "expected" to push those data above the fit line, implies non-linear instrument response behavior during 2004-2006. Hence, we are hesitant to extend the model on the earlier epoch. The implemented forwardprojection approach is justified by the excellent agreement of the long-term and short-term difference spectra, the former implicitly relying on the degradation model, and the latter using data as is.

Correcting the 2007-2013 solar data for instrumental degradation, we assign the overall $0.2 \%$ accuracy to the long-term difference spectra provided in Tables 1 and 2. This assessment is based on the combined influence of the following factors. (1) The scatter within the individual wavelength bins has a typical $\sigma \lesssim 0.1 \%$. This scatter is mainly caused by slight mismatches between the spectra taken at different line-of-sight angles, ideally up to 30 for UV1 and 60 for UV2 and VIS. Note, however, that, due to the impact of the row anomaly (see Appendix A), we only use the data from rows 1-12 in UV1; we also discard rows 1 and 60 both in UV2 and VIS, due to the relatively lower quality of radiometric calibration. Along wavelength, the random scatter in the adjacent wavelength bins (see step 2 above) tends to increase toward the edges of the optical channels, reflecting the gradual loss of consistency in the rowto-row radiometric calibration; we somewhat reduce this scatter by appropriately selecting the cut-off ranges for each particular channel. (2) The uncertainties in the fitting coefficients of the proposed degradation model amount to $\sim 0.1 \%-0.2 \%$ errors, once we compare the data taken around the solar minimum to the current period. (3) We detect $0.1 \%-0.2 \%$ systematic differences in the regions of spectral overlap between different optical channels (e.g., the UV2 and VIS junction at $\lambda=350-360 \mathrm{~nm}$; see the middle panel in Figure 3). These reflect the initial calibration uncertainties inadequately captured by our linear-regression degradation model; we do not attempt to either completely correct or 


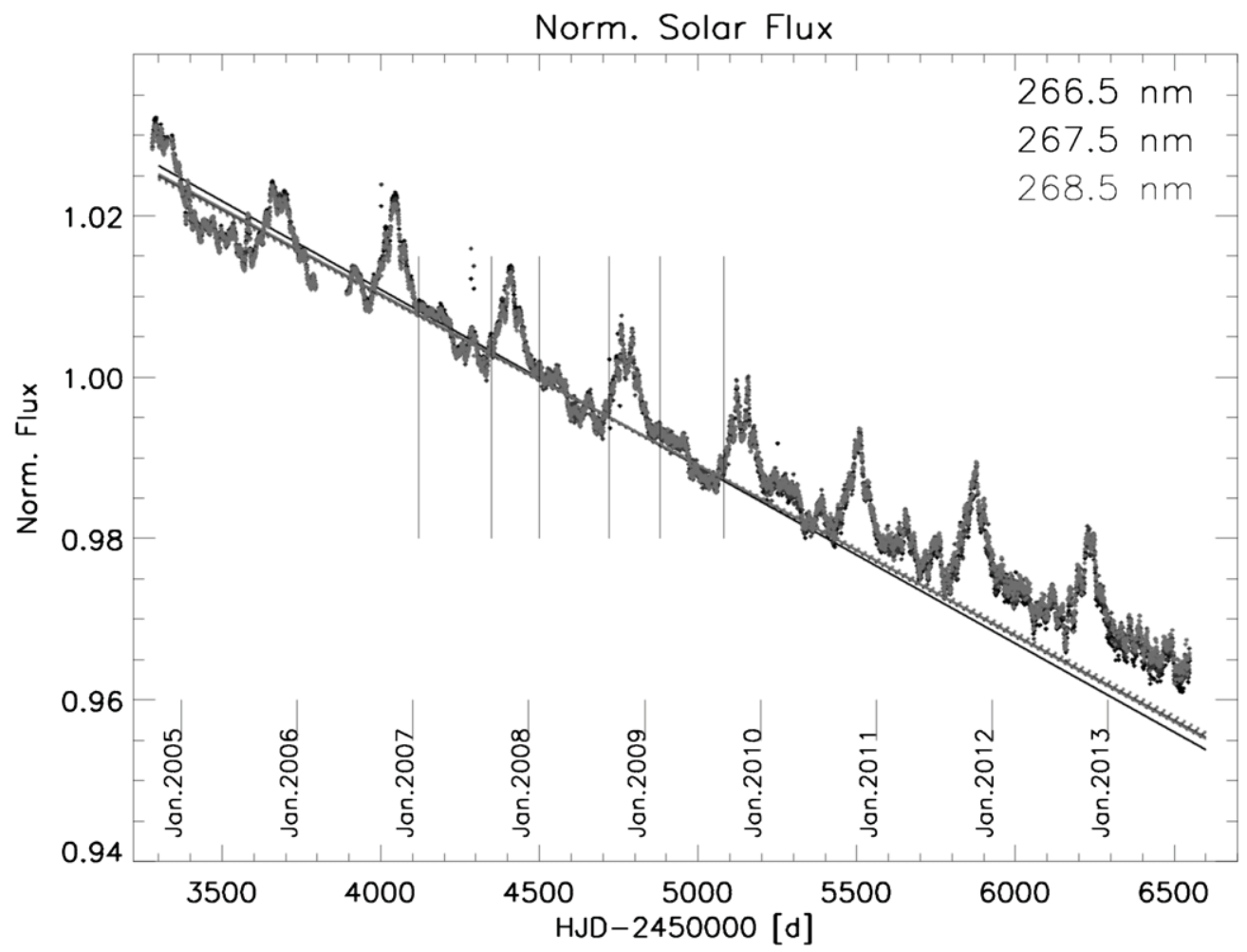

Figure 12. Normalized solar irradiances in the UV1 spectral range at the row 06, used as input for the degradation model. The different colors show the data binned at different wavelengths (provided in the upper right corner). The vertical bars delimit the data chosen for the direct linear fits (full lines), to be compared to the dotted lines which come from the final degradation model. The periods between 2007 November-2008 January and 2008 November-2009 January were excluded from the degradation analysis due to the solar goniometry-related effects.

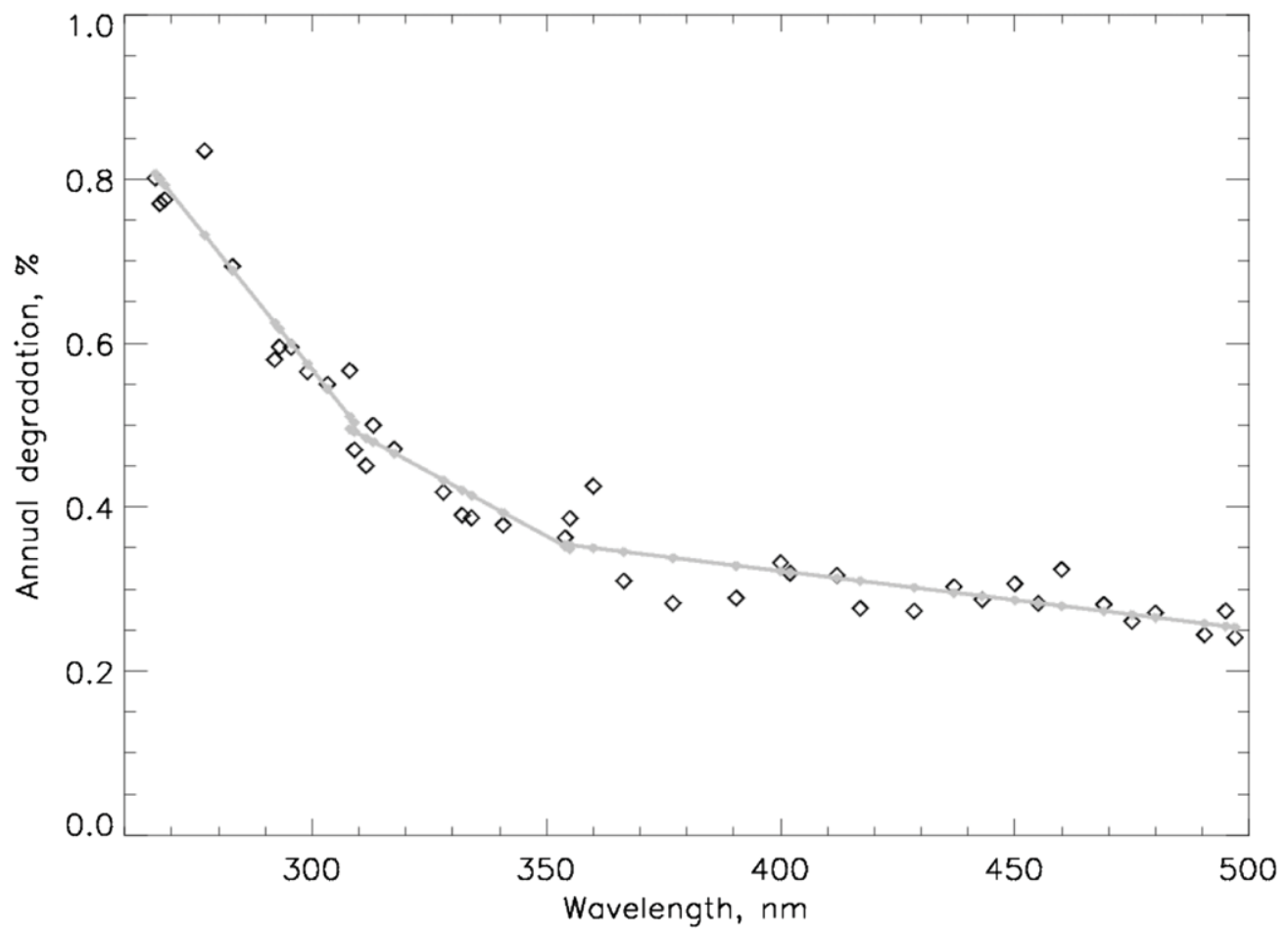

Figure 13. Annual wavelength-dependent degradation rates for the row 06 (UV1) and the corresponding rows 11 (for UV2 and VIS), used as input for the degradation model. The preliminary linear fits are shown as gray lines.

somewhat reduce these mismatches, considering the difficulty of extrapolating their amplitudes on the whole spectral domain; the magnitudes of mismatches prove to be fairly time-independent, down to $\sim 0.1 \%$. (4) We also observe $\sim 0.2 \%$ occasional spikes (see Figure 2) in the time-dependent differences. The spikes are caused by the incompletely compensated fluctuations related to seasonal changes in the solar goniometry. The last factor appears to dominate in the balance of systematic errors. We lessen its influence by (1) calculating the long-term difference spectra on a strictly epoch-related basis, i.e., comparing 2013 June to 2007 
June, 2013 July to 2008 July, etc., and (2) providing the averages of the pair-wise differences coming from different years; e.g., we average the pairs (2013 August-2007 August), (2013 August-2008 August), and (2013 August-2009 August).

The good (to within the assigned uncertainties) match between the short-term and long-term difference spectra (Figure 3), combined with the excellent match between the independently estimated Mg II index (DeLand \& Marchenko 2013) and the wavelength-averaged SSI difference at $\lambda=280.0 \mathrm{~nm}$ (Figure 2), lends extra credibility to the proposed degradation model.

\section{REFERENCES}

Cincunegui, C., Díaz, R. F., \& Mauas, P. J. D. 2007, A\&A, 469, 309 Chandra, S., Lean, J. L., White, O. R., et al. 1995, GeoRL, 22, 2481 Coldewey-Egbers, M., Weber, M., Lamsal, L. N., et al. 2005, ACP, 5, 1015 DeLand, M. T., \& Cebula, R. P. 1993, JGR, 98, 12809

DeLand, M. T., \& Cebula, R. P. 2001, JGR, 106, 21569

DeLand, M. T., \& Cebula, R. P. 2008, JGRA, 113, A11103

DeLand, M. T., \& Cebula, R. P. 2012, JASTP, 77, 225

DeLand, M., \& Marchenko, S. 2013, JGRD, 118, 3415

Dobber, M. R., Dirksen, R. J., Levelt, P. F., et al. 2006, ITGRS, 44, 1209

Dobber, M. R., Kleipool, Q., Dirksen, R., et al. 2008, JGR, 113, D15S06

Ermolli, I., Criscuoli, S., Centerone, M., Giorgi, F., \& Penza, V. 2007, A\&A, 465,305

Ermolli, I., Matthes, K., Dudok de Wit, T., et al. 2013, ACP, 13, 3945

Fontenla, J. M., Harder, J., Livingston, W., Snow, M., \& Woods, T. 2011, JGR, 116, D20108

Fontenla, J., Reichmann, E. J., \& Tandberg-Hanssen, E. 1988, ApJ, 329, 464

Fröhlich, C. 2009, A\&A, 501, L27

Fröhlich, C., \& Lean, J. 2004, A\&ARv, 12, 273

Gomes da Silva, J., Santos, N. C., Bonfils, X., et al. 2012, A\&A, 541, 9

Grossmann-Doerth, U. 1994, A\&A, 285, 1012
Harder, J. W., Fontenla, J. M., Pilewskie, P., Richard, E. C., \& Woods, T. N. 2009, GeoRL, 36, L07801

Heath, D. F., \& Schlesinger, B. M. 1986, JGR, 91, 8672

Jaross, G., \& Warner, J. 2008, JGR, 113, D16S34

King, J. H., \& Papitashvili, N. E. 2005, JGRA, 110, A02104

Kopp, G., \& Lean, J. L. 2011, GeoRL, 38, L01706

Lean, J. L., \& DeLand, M. T. 2012, JCli, 25, 2555

Lean, J. L., \& Woods, T. N. 2012, in Heliophysics: Evolving Solar Activity and the Climates of Space and Earth, ed. C. J. Schrijver \& G. L. Siscoe (Cambridge: Cambridge Univ. Press), 269

Lean, J. L., Rottman, G. J., Kyle, H. L., et al. 1997, JGRD, 102, 29939

Levelt, P. F., van den Oord, G. H. J., Dobber, M. R., et al. 2006, ITGRS, 44, 1093

Livingston, W., Wallace, L., White, O. C., \& Giampapa, M. S. 2007, ApJ, 657, 1137

Meunier, N., \& Delfosse, X. 2009, A\&A, 501, 1103

Mitchell, W. E., Jr., \& Livingston, W. C. 1991, ApJ, 372, 336

Pagaran, J., Weber, M., \& Burrows, J. 2009, ApJ, 700, 1884

Penza, V., Pietropaolo, E., \& Livingston, W. 2006, A\&A, 454, 349

Przybilla, N., \& Butler, K. 2004, ApJL, L61

Robertson, P., Endl, M., Cochran, W. D., \& Dodson-Robinson, S. E. 2013, ApJ, 764, 3

Rottman, G. 2005, SoPh, 230, 7

Santos, N. C., Gomes da Silva, J., Lovis, C., \& Melo, C. 2010, A\&A, 511,54

Swartz, W. H., Stolarski, R. S., Oman, L. D., Fleming, E. L., \& Jackman, C. H. 2012, ACP, 12, 5937

Tapping, K. F. 1987, JGR, 92, 829

Thuillier, G., Floyd, L., Woods, T. N., et al. 2004, in Geophysical Monograph 141, Solar Variability and its Effects on Climate, ed. J. M. Pup et al. (Washington, DC: AGU Geophysical Monograph Series), 171

Thuillier, G., Melo, S. M. L., Lean, J., et al. 2014, SoPh, 289, 1115

Wallace, L., Hinkle, K. H., Livingston, W. C., \& Davis, S. P. 2011, ApJS, 195,6

White, O. R., Livingston, W. C., \& Wallace, L. 1987, JGR, 92, 823

Woods, T. N., Tobiska, W. K., Rottman, G. J., \& Worden, J. R. 2000, JGR, 105 , 27195 